\title{
Empirisches Vorgehen und Methodik
}

In diesem Kapitel wird das methodische Vorgehen, das im empirischen Teil dieser Arbeit (Kp. 7 und Kp. 8) zum Einsatz kommt, transparent gemacht und begründet. Ziel der Empirie ist es, die Datengrundlage für die Beantwortung der Forschungsfrage , Wie entstehen Themen in der Planungswissenschaft' herzustellen. Hierfür leitet der analytische Rahmen ein Kategoriengerüst aus der Theorie ab, das den Fokus auf die potenziell erklärungsrelevanten Aspekte richtet. Die Methodik muss ermöglichen, diese Aspekte im Zuge der empirischen Analyse zu erfassen. Als Untersuchungsfeld dient die deutsche Planungswissenschaft. Hier sind Themenkarrieren nachzuweisen, zu rekonstruieren und Mechanismen der wissenschaftlichen Aufmerksamkeitsentstehung offenzulegen.

Themenkarrieren in der Planungswissenschaft werden durch die aggregierten Themenentscheidungen der Planungswissenschaftler*innen konstituiert. Diese Entscheidungen stehen deshalb im Zentrum der empirischen Untersuchung. Es werden dabei, dem Programm der institutionalistischen Wissenschaftssoziologie folgend, die personenunabhängigen Regeln und Normen zur Erklärung in den Vordergrund gerückt. Die Beschaffenheit des institutionellen Rahmens der Planungswissenschaft wird folglich als Erklärungsmoment für das kollektive Handeln der Planungswissenschaftler*innen und damit für die Entstehung von Aufmerksamkeit gegenüber Themen anvisiert.

Es wird ein vergleichender Fallstudienansatz gewählt, wobei die beiden Themenkarrieren Schrumpfende Städte und Klimawandel im Zeitraum 1995-2014 als Fälle dienen. Der Beginn des Untersuchungszeitraums wurde auf der Grundlage einer Voruntersuchung ausgewählt, die ergab, dass beide Themen erst nach 1995 in der Planungswissenschaft etabliert worden sind. Das Ende des Untersuchungszeitraums (Dezember 2014) markiert gleichzeitig den Beginn der Datenaufnahme (Januar 2015). Die Analyse kann nur ex post erfolgen, weil Themenkarrieren

A. Gravert, Themenkarrieren in der Wissenschaft, 
erst nach der erstmaligen Etablierung des Themas als solche identifizierbar sind. Folglich muss auf Verfahren der Datenerhebung abgestellt werden, mit denen vergangene Prozesse möglichst detailliert nachvollzogen werden können.

Um diese empirische Rekonstruktion leisten zu können, wird ein mixed methods Ansatz angewandt. Dieser folgt dem von Pluye und Quan Nha Hong (2013: 30) formulierten Leitbild: „mixing methods combines the power of stories and the power of numbers". Die Verwendung sowohl quantitativer als auch qualitativer Verfahren gewährleistet nicht nur die notwendige empirische Evidenz. Vielmehr werden im Rahmen dieses Vorhabens die Methoden kombiniert und integriert, sodass qualitative Schritte quantitative Ergebnisse erst ermöglichen und umgekehrt. Quantitative Daten dienen der Identifizierung der Themenkarrieren und der Deskription des Verlaufs der Aufmerksamkeit gegenüber den Themen. Durch bibliometrische Kennziffern soll dargelegt werden, wie sich die beiden Themen in der Planungswissenschaft manifestiert haben. Eine formale Netzwerkanalyse zeigt die Eigenschaften themenspezifischer Netzwerke von Planungswissenschaftler*innen und planungswissenschaftlichen Veröffentlichungen. Aus der Theorie abgeleitet werden Netzwerkpositionen (bspw. zentrale Akteur*innen) definiert, die anschließend einer qualitativen Analyse unterzogen werden. Schließlich werden durch die Netzwerkanalyse Akteur*innen identifiziert, die diese Netzwerkpositionen bekleiden. Die ausgewählten Akteur*innen werden jeweils im Rahmen eines leitfadengestützten Interviews befragt. Zur Vorbereitung des Interviews werden wiederum sowohl quantitative (bspw. Zitationskennziffern) als auch qualitative (bspw. Forschungsbiografien) personenund forschungsbezogene Daten verwandt. Über eine grafische Darstellung werden die Akteur*innen mit ihren persönlichen Daten bzw. ihrer persönlichen Forschungsbiografie konfrontiert.

Im Folgenden wird das mehrstufige empirische Forschungsdesign erläutert. Zunächst ist dabei herzuleiten, warum ein vergleichender Fallstudienansatz gewählt wird und anhand welcher Kriterien die beiden Fälle ausgewählt werden (Kp. 6.1). Im Anschluss wird die Operationalisierung der beiden gewählten Themen vorgenommen (Kp. 6.2). In Abschnitt 6.3 wird die quantitative Analyse erläutert, was die Datenerhebung sowie die angewandten Auswertungsmethoden der Bibliometrie und der formalen Netzwerkanalyse mit einschließt. AbschlieBend wird in Abschnitt 6.4 auf die qualitative Datenerhebung und -auswertung eingegangen, wobei primär leitfadengestützte Interviews zum Einsatz kommen. 


\subsection{Fallstudienansatz, Auswahl und Abgrenzung der Fälle}

Zur Beantwortung der Frage wird ein multipler Fallstudienansatz mit der vergleichenden Untersuchung von zwei Fällen gewählt. Dieser eignet sich speziell für die Analyse besonders komplexer organisationaler Zusammenhänge (Yin 2009: 4), die Gegenstand der vorliegenden Untersuchung sind. Fallstudien erfassen und analysieren zeitgenössische soziale Prozesse in einem realitätsnahen Kontext, insbesondere wenn die Grenzen zwischen Phänomen und Kontext nicht offensichtlich sind (ebd.: 18). Sie ermöglichen es, komplexe Dynamiken unter Einbeziehung kontextabhängigen Wissens in einer dichten Beschreibung nachzuvollziehen. Darüber hinaus eignen sich Fallstudien dazu, unterschiedliche Variablen und Datenarten theoriegeleitet zu erschließen und in einen aufschlussreichen Zusammenhang einzuordnen (Günzel 2016a: 143; Flyvbjerg 2006). Gemäß Yin (2009: 61) ist es, sofern eine Wahlmöglichkeit besteht, grundsätzlich vorzuziehen mehrere Fallstudien durchzuführen, um die Wahrscheinlichkeit einer, geeigneten ' Fallstudie zu erhöhen und weil ein substanzieller analytischer Mehrwert bei dem Vergleich zweier Fallstudien erwartet werden kann. Dagegen nimmt der erwartete Mehrwert bei einer dritten Fallstudie (oder weiterer) tendenziell ab, sodass einem steigenden Arbeitsaufwand ein abnehmender Grenznutzen gegenübersteht - insbesondere dann, wenn eine Ähnlichkeit der Fälle konstatiert werden kann.

Die beiden Fälle werden exemplarisch untersucht. Nicht die Analyse der Themen selbst steht also im Fokus der Auswertung, sondern die dahinterliegenden sozialen Strukturen und Mechanismen einschließlich deren Veränderung im Verlauf der Themenkarriere. Als Fall gilt in dieser Arbeit eine Themenkarriere, also der Verlauf der fachöffentlichen Aufmerksamkeit gegenüber einem Thema in der Planungswissenschaft. Der erstmalige, textlich manifestierte Beitrag zum Thema markiert den Beginn der Themenkarriere. Dementsprechend würde das Ende der Themenkarriere durch den letzten Beitrag markiert, der sich, solange die Planungswissenschaft existiert, nicht bestimmen lässt. Es können auch Ereignisse vor dem Beginn der Themenkarriere in die Untersuchung einfließen, sofern sich ein Einfluss auf die Themenkarriere vermuten lässt.

Für die Auswahl der Fallstudien bzw. der Themen lassen sich aus der Forschungsfrage, dem Untersuchungsgegenstand und der gewählten Methodik wesentliche Bedingungen ableiten. Zunächst müssen die Themen jeweils eine Themenkarriere in der Planungswissenschaft durchlaufen (haben). Das heißt, dass zumindest die Latenzphase und die anschließende Etablierung des Themas 
über einen gewissen Zeitraum hinweg beobachtbar sein müssen, denn Untersuchungsgegenstand ist die tatsächliche Themenkarriere bzw. ihre Entstehung. Dass einige Themen (bisher) keinen Durchbruch in der Fachöffentlichkeit erreicht haben und warum das so ist, ist dagegen nicht Gegenstand dieser Untersuchung. Des Weiteren müssen sich die Themen durch eine hohe Wirkmächtigkeit auszeichnen: Zum einen impliziert der Untersuchungsgegenstand der Themenkarriere an sich schon die Notwendigkeit einer gewissen Etablierung im fachöffentlichen Diskurs. Zum anderen sollte das Thema in den zentralen Institutionen der Planungswissenschaft (bspw. Fördermittelvergabe) bereits eine messbare Wirkung entfaltet haben. Darüber hinaus verlangt die vorgesehene Netzwerkanalyse eine genügend große Grundgesamtheit an Veröffentlichungen in den verschiedenen Fachzeitschriften der Planungswissenschaft. Schließlich muss das Thema gegenwärtig sein, denn für die Untersuchung der Themenkarriere muss auf die beteiligten Akteur*innen (bspw. Vertreter*innen aus Forschung, Gremien, Fördermittelvergabe) für Interviews zugegriffen werden können. Für die Qualität der Interviews sollte der zeitliche Abstand zu den besprochenen Erfahrungen gering gehalten werden, da bspw. individuelle Einschätzungen zu Themenkarrieren oder Akteur*innennetzwerken sich mit zeitlichem Abstand und mit Veränderung der Position im Netzwerk wandeln.

Anhand dieser Kriterien wurden die Themen Schrumpfende Städte und Klimawandel als Fälle ausgewählt. Sie sind vergleichbar, indem sie in erster Linie Inhalte der Planung transportieren. Es werden dabei vor allem materielle Anpassungserfordernisse adressiert und erst in zweiter Linie Theorien und Methoden der Planung. Die Themenkarrieren haben deshalb unmittelbar Einfluss auf planungspraktische Handlungsoptionen sowie letztlich auf die Gestaltung von Räumen. Des Weiteren erscheint der Verlauf beider Themenkarrieren mit signifikanten Aufmerksamkeitsspitzen in den 2000er Jahren zeitlich weitgehend entkoppelt von der Entwicklung vermeintlich objektiver Handlungserfordernisse im Kontext der bereits Jahrzehnte zuvor und wahrscheinlich auch noch Jahrzehnte danach raumrelevanten Themen. Diese Entkopplung ermöglicht einen freien Blick auf planungswissenschaftsinterne, soziale Mechanismen der Themenkarriere. Beide Themen bieten zudem starke Anknüpfungspunkte an gesamtgesellschaftliche Diskurse, wobei das Thema Klimawandel in besonderem Maße fremdreferenziell erscheint, während das Thema Schrumpfende Städte auf den ersten Blick stärker durch die Planungswissenschaft selbst geprägt wurde (Gravert et al. 2013: 23-25).

Die beiden gesamtgesellschaftlichen Megathemen Schrumpfende Städte und Klimawandel, genauso wie die anwendungsorientierte Planungswissenschaft, 
stellen sicherlich wissenschaftssoziologische Sonderfälle dar. Bei der Kontextualisierung der Ergebnisse in den Kanon der Wissenschaftssoziologie und der Planungswissenschaft sind daher die besonderen disziplinären und thematischen Charakteristika zu berücksichtigen. Zu der Frage, warum Sonderfälle für die vorliegende Untersuchung gewählt wurden, ist erstens zu erwidern, was denn der diesen Sonderfällen gegenüberzustellende ,Normalfall ' wäre. Weder die im Rahmen dieser Arbeit evaluierten empirischen Studien (siehe insb. Kp. 2) noch der Blick auf andere Disziplinen oder andere planungswissenschaftliche Themen legen Beispiele nahe, die als ,Normalfälle ' bezeichnet werden könnten. Weiterhin ist zu bemerken, dass allein die Exposition der betrachteten Gegenstände keine Aussage über den zu erwartenden Erkenntnisgewinn zulässt.

\subsection{Operationalisierung und Abgrenzung der Themen}

Bei der Datenerhebung und -auswertung ist die Abgrenzung und Operationalisierung der Themen Schrumpfende Städte und Klimawandel ein neuralgischer Punkt. Die hier vorgenommenen Entscheidungen konstruieren erst den Untersuchungsgegenstand dieser Forschungsarbeit. Die Operationalisierung wird für die Themenzuordnung der 7.053 Artikel in planungswissenschaftlichen Zeitschriften im Zeitraum 1995-2014 eingesetzt (siehe Kp. 6.3.2). Das konkrete Ziel der Operationalisierung ist die intersubjektiv überprüfbare Identifizierung einschlägiger planungswissenschaftlicher Fachbeiträge. Einschlägig heißt, die Fachbeiträge werden von der planungswissenschaftlichen Gemeinschaft dem zu untersuchenden Thema zugeordnet. Aus den in Abschnitt 5.1 zusammengefassten Merkmalen von Themen ergeben sich dafür verschiedene Herausforderungen der Operationalisierung:

- Abgrenzung: Ein Thema hat Schnittmengen mit anderen Themen, vereint verschiedene Subthemen zu einem Sinnkomplex und ist seinerseits als Subthema Teil verschiedener übergeordneter Themen. Das wesentliche Problem dabei ist die Abgrenzung: Ab wann gehört ein Beitrag zum Thema?

- Signifikation: Linguistisch betrachtet ist ein Thema ein Sinnkomplex (Signifikat), der durch ein Netzwerk mit Bedeutung aufgeladener Begriffe (Signifikanten) repräsentiert wird. Ähnliche Sinnkomplexe sind bei unterschiedlichen Akteur*innen, zu unterschiedlichen Zeitpunkten aber mit unterschiedlichen Begriffen verbunden. Zudem kann die Begriffswahl strategisch motiviert sein. So werden etwa die Begriffe ,Verdrängung ' bzw. ,Aufwertung' in bestimmten Diskursen von bestimmten Akteur*innengruppen strategisch vermieden 
bzw. bevorzugt, selbst wenn auf dasselbe stadtentwicklungspolitische Ereignis Bezug genommen wird.

- Intersubjektivität: Es gibt so viele Verständnisse eines Themas, wie es Teilnehmende der Kommunikation zu dem Thema gibt. Zwar lässt sich von einem geteilten Themenverständnis sprechen, wie aber erhebt man dieses? Was sind die Schnittmengen? Die Abgrenzung einzelner Themen sollte möglichst nicht willkürlich von dem*der Forschenden vorgenommen werden, sondern von dem Forschungsgegenstand selbst, also von den Beiträgen der untersuchten Planungswissenschaftler*innen, ausgehen.

- Asynchronie: Die vorliegende Untersuchung beobachtet Themen über einen Zeitraum von 20 Jahren. Ein Thema transformiert sich über die Zeit, ist also zu unterschiedlichen Zeitpunkten nicht-identisch mit sich selbst. Ein Thema erzeugt bei den gleichen Personen im Jahr 1995 andere Sinnkomplexe als im Jahr 2014. Hinzu kommt, dass sich der Kreis der Personen, der sich mit einem Thema befasst, verändert.

Die im Folgenden erläuterte Methodik muss diese Punkte weitestgehend auffangen, wobei ein Schwerpunkt auf die intersubjektive Nachvollziehbarkeit gelegt wird. Da sich die benannten Herausforderungen jedoch nicht ohne Einschränkungen im Zuge der Datenerhebung bewältigen lassen, sind sie auch in der Datenauswertung zu berücksichtigten.

Für die themenspezifische Datenauswertung werden all jene Artikel als einschlägig identifiziert, die eine explizite Kontextualisierung im jeweiligen Thema enthalten. Für eine solche Kontextualisierung kann bereits eine Überschrift oder ein einzelner Absatz genügen. Andererseits wird eine Kommunikation erst dann Teil der Themenkarriere, wenn sie in dem jeweiligen Thema gerahmt wird. Zwei Artikel können also eine nahezu identische Fragestellung aufweisen, durch die Rahmung in unterschiedlichen Kontexten ist es jedoch möglich, dass einer der beiden Artikel als einschlägig befunden wird, der andere aber nicht.

Zur Auswahl einschlägiger Artikel aus den planungswissenschaftlichen Zeitschriften im Untersuchungszeitraum wird ein mehrstufiges Verfahren angewendet, das ein größtmögliches $\mathrm{Maß}$ an Objektivität (bzw. intersubjektiver Nachvollziehbarkeit), Reliabilität und Validität gewährleistet. Als methodologischer Ausgangspunkt für die Themenzuordnung werden Signifikanten bzw. Schlüsselbegriffe gewählt. Neben „Klimawandel“, „Klimaerwärmung“ und „Schrumpf*“ wird hierfür auch der Begriff „Stadtumbau“ genutzt, der mit wenigen Ausnahmen im Schrumpfungskontext beheimatet ist.

Die folgenden vier Schritte werden für die Auswahl einschlägiger Artikel befolgt (siehe Abb. 6.1): 
Computergestützte Prüfung des Titels: Enthält der Titel einen gesuchten String

( ${ }^{*}$ klimawandel*, *klimaerwärmung*, *schrumpf*, oder *stadtumbau*)?

$\downarrow$ ja $\quad \downarrow$ nein

Händische Prüfung des Titels: Handelt es sich um kein Falsch Positives?

Händische Prüfung des Titels: Könnte der Artikel einschlägig sein?

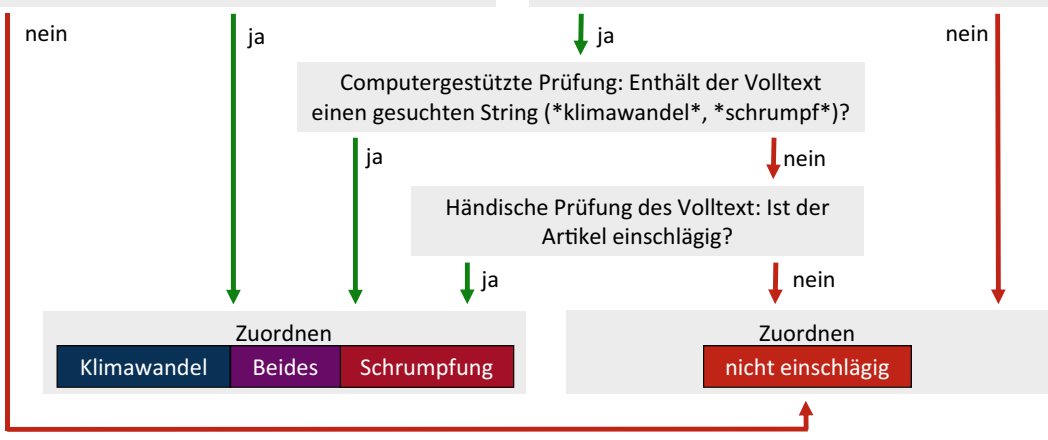

Abb. 6.1 Auswahl einschlägiger planungswissenschaftlicher Artikel (Eigene Darstellung)

1. In einem ersten Schritt wird der Titel überprüft, wobei der Begriff Titel in dieser Arbeit immer auch den Untertitel, sofern vorhanden, mit einschließt. Artikel, die die Zeichenketten *klimawandel*, *klimaerwärmung*, *schrumpf* und/oder *stadtumbau* im Titel tragen, werden händisch um mögliche Falsch Positive (bspw. „Schrumpfende Einnahmen der Kommunen“ enthält den gesuchten String, ist aber nicht unbedingt einschlägig) reduziert und schließlich als einschlägig eingestuft.

2. Aus den verbleibenden Titeln wird eine Auswahl potenziell einschlägiger Artikel getroffen. Diese Auswahl wird großzügig angelegt, sodass das Übersehen einschlägiger Artikel vermieden wird, andererseits jedoch viele nicht einschlägige Artikel Teil der Auswahl werden. Als Hilfestellung für die Vorauswahl dient eine Liste mit 18 Hinweiswörtern (bspw. *bevölkerungsrückgang*). Allerdings werden auch Titel, die kaum Rückschlüsse auf den Inhalt zulassen (bspw. „Letzter macht das Licht aus“), in diesem zweiten Schritt berücksichtigt.

3. Im dritten Schritt wird die entstandene Auswahl potenziell einschlägiger Artikel einer Volltextsuche unterzogen: Artikel, die die Zeichenketten *klimawandel*, *klimaerwärmung*, *schrumpf* und/oder *stadtumbau* enthalten, werden direkt als einschlägig eingestuft. 
4. In einem vierten Schritt werden die verbliebenen, potenziell einschlägigen Artikel unter Berücksichtigung der 18 Hinweiswörter händisch gesichtet und gegebenenfalls als einschlägig eingestuft. Generell ist hierbei ein Mindestkriterium die Thematisierung demografischer Schrumpfung oder globaler Klimaerwärmung als zentraler Bestandteil des Artikels.

In der weiteren Analyse beider Themenkarrieren wird die Einstufung der Einschlägigkeit eines Artikels mehrmals auf Basis unterschiedlicher Indikatoren überprüft. So würde bspw. eine hohe bibliografische Kopplung (Ähnlichkeit der Literaturlisten verschiedener Artikel) zwischen als nicht-einschlägig und als einschlägig eingestuften Artikeln auffallen.

Insgesamt wurden nach diesem Verfahren aus 7.053 Artikeln 325 dem Thema Schrumpfende Städte (siehe Kp. 7) und 337 dem Thema Klimawandel (siehe Kp. 8) zugeordnet. 12 Artikel wurden beiden Themen zugeordnet.

\subsection{Quantitative Analyse: Bibliometrie und Netzwerkanalyse}

Die quantitative Erhebung und Auswertung stellt die Analyse der beiden Themenkarrieren auf ein Fundament aus möglichst objektiven, reliablen und validen Informationen. Zum einen wird der Verlauf der planungswissenschaftlichen Aufmerksamkeit gegenüber beiden Themen gemessen und dargestellt (bspw. anhand der Anzahl einschlägiger Artikel pro Jahr). Zum anderen wird die soziale Dynamik dieses Verlaufs mit netzwerkanalytischen Auswertungen nachvollzogen (bspw. anhand der Veränderungen der Kooperationsstruktur der Planungswissenschaftler*innen). Weiterhin wird die qualitative Analyse durch quantitative Daten unterstützt: Einerseits erfolgt die Auswahl der Akteur*innen für die Leitfaden-Interviews mit Hilfe quantitativer Abfragen, andererseits werden die Interviews auf der Grundlage personenbezogener Kooperations-, Zitations- und forschungsinhaltlicher Auswertungen geleistet.

Die Grundlage für die quantitative Analyse wissenschaftlicher Prozesse und Strukturen sind wissenschaftliche Publikations- und Zitationsdaten. Dies macht sie zu einer bibliometrischen Analyse. Zentrale Annahmen und Konzepte der Bibliometrie werden deshalb in Abschnitt 6.3.1 erläutert. Im Anschluss wird die quantitative Erhebung sowie die Aufbereitung der Publikations- und Zitationsdaten geschildert. Im Rahmen der bibliometrischen Datenauswertung kommen Methoden und Konzepte der deskriptiven Statistik sowie der Netzwerkanalyse zum Einsatz. Die deskriptiv statistischen Auswertungen dieser Arbeit zielen 
auf Häufigkeiten, Summen, Mittelwerte und Varianzen (bspw. ,wieviele einschlägige Artikel werden pro Jahr veröffentlicht?'). Sie bedürfen keiner weiteren methodologischen Erläuterungen. Dagegen ist die netzwerkanalytische Auswertung voraussetzungsreich, weshalb deren grundlegende Annahmen, Konzepte und Methoden in Abschnitt 6.3.3 dargelegt werden. Am Ende dieses Teilkapitels werden in Form eines Glossars einige Begriffe und Analyseparameter der (bibliometrischen) Netzwerkanalyse erklärt, die im Verlauf der empirischen Kapitel (7 und 8) verwendet werden.

\subsubsection{Grundlagen der Bibliometrie}

Die Wissenschaft wird in dieser Arbeit als ein soziales System betrachtet, in welchem Wissenschaftler*innen Erkenntnisse in einem fachgemeinschaftlichen Forschungsprozess und auf der Basis eines geteilten Wissensstandes gewinnen. Wissenschaftliche Wissensproduktion ist folglich immer das Ergebnis von Kommunikation, nie das Werk isolierter Forscher*innen. Verschiedene Akteur*innen beziehen Ideen und Wissen voneinander, verfassen gemeinsam Publikationen und begutachten das Werk der anderen. Die Veränderung der fachgemeinschaftlichen Wissensstruktur sowie die Zuweisung von Reputation werden als das Produkt sozialer und intellektueller Beziehungen verstanden, die sich bspw. in Form von Ko-Autorenschaften und Zitationen manifestieren.

Der Prozess der gemeinschaftlichen Wissensproduktion lässt sich mit den Methoden der Bibliometrie abbilden und analysieren (Aufenvenne und Steinbrink 2014: 263). Als Teil der Szientometrie, also der quantitativen Analyse von Wissenschaft, wählt die Bibliometrie Publikationen zum Ausgangspunkt der Analyse (Broadus 1987: 377). Bibliometrische Methoden stellen ein wichtiges Analyseinstrument kritischer Wissenschaftsbeobachtung dar, das weit über die seit einigen Jahren an Bedeutung gewinnende Bewertung wissenschaftlichen Outputs anhand quantifizierbarer Indizes hinausgeht (Aufenvenne und Steinbrink 2014: 263).

Die bibliometrische Analyse wissenschaftlicher Publikationen nimmt für die hier vorgenommene empirische Beobachtung von Themenkarrieren in der Planungswissenschaft eine zentrale Rolle ein. In diesen Publikationen findet die formalisierte Kommunikation über die Wissensproduktion statt. Zwar sind auch informelle Kommunikationen (bspw. Gespräche oder Emails) mitunter von großer Bedeutung für die wissenschaftliche Erkenntnisgewinnung, allerdings nur, wenn sie sich langfristig auch in Publikationen manifestieren. So argumentieren Morris und Van der Veer Martens (2008: 219): 
„[...] the nature of science is such that, without the published papers, the informal communication relations of most scholars appear of limited interest. Although scientific progress cannot be achieved without informal communication, scientific progress can not be verified without formal communication. "

Weingart (2003: 32) geht deshalb soweit, nur in Publikationen veröffentlichte Ergebnisse zum wissenschaftlichen Wissen zu zählen. In dieser Arbeit genügt es, Publikationen als das entscheidende Medium für die fachöffentliche Kommunikation und das Festhalten wissenschaftlicher Erkenntnisse sowie dahinterliegender Entstehungszusammenhänge aufzufassen. Insbesondere in Fachzeitschriften veröffentlichte Artikel stellen seit den Anfängen der modernen Wissenschaft das zentrale Produkt wissenschaftlicher Arbeit dar (ebd.). Hier werden Forschungsergebnisse zwischen Mitgliedern der Fachgemeinschaften besonders intensiv rezipiert und der kollegialen Kritik unterworfen.

Im Zuge der bibliometrischen Analyse werden zwei Arten von Beziehungen untersucht: Ko-Autorenschaften und Zitationen. Ko-Autorenschaften sind Beziehungen die sich aus gemeinsam verantworteten Publikationen ergeben und denen in der Regel vielfältige formale und informelle Formen der Zusammenarbeit vorausgehen (Heinze 2012: 192). Sie dienen als Indikator für wissenschaftliche Kooperation und Kommunikation, die in der Regel eine persönliche Beziehung, eine intensive Interaktion sowie eine gewisse intellektuelle Nähe zwischen den Autor*innen voraussetzt (Arzheimer und Schoen 2009: 607; Steinbrink et al. 2010: 12). Indem Ko-Autorenschaften Kollaborationen indizieren, handelt es sich um soziale Beziehungen. Im Gegensatz dazu deuten Zitationen primär auf intellektuelle Relationen zwischen zitierender und zitierter Quelle. Dieses Verständnis der Zitation als textliche Spur einer Wissensbeziehung bzw. als sichtbares Element eines diachronen Wissensnetzwerks ergibt sich aus ihrer formalen Funktion (Offenlegung der Quellen). Allerdings ist auch die latente Funktion (Verteilung sozialer Anerkennung auf andere Autor*innen) zu beachten (siehe Kp. 5.3) (Arzheimer und Schoen 2009: 606-607; Steinbrink et al. 2010: 11). Ähnlich wie bei der Ko-Autorenschaft, die nur einen Teil der Kooperationsbeziehungen erfasst, handelt es sich bei Zitationen um besonders sichtbare Bezugnahmen auf bereits publiziertes Wissen, die bei Weitem nicht alle faktischen intellektuellen Bezüge einer Publikation einschließlich des jeweils vorausgehenden Forschungsprozesses miteinbeziehen (Heinze 2012: 192). 


\subsubsection{Erhebung und Aufbereitung der Daten}

Ausgangspunkt der quantitativen Analyse ist die systematische Erhebung der Artikel in den deutschsprachigen planungswissenschaftlichen Zeitschriften im Untersuchungszeitraum 1995 bis 2014. Diese Eingrenzung der Datengrundlage ermöglicht eine konsistente Erhebung sowie eine vergleichsweise hohe Repräsentativität bei vertretbarem Aufwand. Weitere Publikationsmedien deutschsprachiger Planungswissenschaftler*innen systematisch zu erheben - beispielsweise in den unübersichtlichen und nicht systematisch katalogisierten Publikationssegmenten der Monografien und Sammelbände - wäre empirisch nicht leistbar (vgl. Aufenvenne und Steinbrink 2014: 267). Eine zusätzliche Erhebung von englischsprachigen oder in anderen Disziplinen verorteten Zeitschriften würde unter anderem das Filtern auf Beiträge deutscher Planungswissenschaftler*innen sowie die sprachliche Anpassung der Themenabgrenzung erfordern. Diesem Aufwand steht ein geringer Nutzen entgegen, da fraglich ist, inwieweit solche Zeitschriften als Kommunikationsplattform für deutschsprachige, planungswissenschaftliche Diskurse gelten können.

Der Konzentration auf planungswissenschaftliche Artikel liegt außerdem die Annahme zugrunde, dass diese einen repräsentativen Querschnitt des Erkenntnisgewinns der Disziplin darstellen. Schließlich sind Zeitschriften das zentrale Medium für das wissenschaftliche Kommunikationssystem, das heißt für das gegenseitige Wahrnehmen und Rezipieren sowie für die Zuschreibung von Reputation (Weingart 2003: 32-34). Resonanter Erkenntnisgewinn wird deshalb in der Regel (auch) in Artikeln durch die Urheber*innen der Fachöffentlichkeit vorgestellt oder zumindest von Dritten besprochen. Dies gilt für Beiträge in anderen Publikationsformaten (bspw. Monografien) sowie in disziplinär und sprachlich anders zugeschnittenen Zeitschriften gleichermaßen. Ferner werden durch die miterhobenen Zitationen nachvollziehbare Bezüge zu den grundlegenden Publikationen in den Zeitschriftenartikeln kenntlich gemacht. Indem alle in den einschlägigen Artikeln getätigten Zitationen systematisch erfasst werden, können wichtige Veröffentlichungen unabhängig vom Publikationsorgan bzw. -segment ermittelt werden. Veröffentlichungen, die sich etwa durch besonders häufige oder besonders grundlegende inhaltliche Bezüge aus der Artikelauswahl hervorheben, die aber nicht Teil des Zeitschriftenkorpus sind, werden nachträglich in die Analyse einbezogen.

Im Zuge der quantitativen Analyse planungswissenschaftlicher Artikel werden der Volltext, die in den Artikeln getätigten Zitationen sowie die Metadaten, das heißt insbesondere Autor*innen, Titel, Erscheinungsjahr und Zeitschriftenausgabe erhoben. Artikel, deren Autorenschaft nicht gekennzeichnet ist, sind nicht 
Bestandteil der Analyse. Aus forschungspragmatischen Erwägungen, die sich aus der unterschiedlich gelagerten Datenverfügbarkeit sowie aus der unterschiedlichen Funktion der Zeitschriften im Rahmen der planungswissenschaftlichen Wissensproduktion ergeben, werden die Daten der verschiedenen Zeitschriften in unterschiedlicher Tiefe erhoben (siehe Tab. 6.1). Die folgenden Zeitschriften werden systematisch erfasst:

- disP - The Planning Review (im Folgenden disP) (699 Artikel im Untersuchungszeitraum)

- Informationen zur Raumentwicklung (IzR) (1.421 Artikel)

- Forschungs- und Sitzungsberichte bzw. seit 2013 Forschungsberichte der Akademie für Raumforschung und Landesplanung (ARL-FB) (587 Artikel)

- PLANERIN (1.867 Artikel)

- RAUM (2012 eingestellt) (909 Artikel)

- RaumPlanung (803 Artikel)

- Raumforschung und Raumordnung (RuR) (767 Artikel)

Somit sind alle wesentlichen deutschsprachigen planungswissenschaftlichen Zeitschriften des Untersuchungszeitraums Teil der Untersuchung mit Ausnahme der ,fub - Flächenmanagement und Bodenordnung ', bei der keine Datenfreigabe möglich war. Die ebenfalls in Frage kommende ,pnd online" scheidet aufgrund der Unregelmäßigkeit und des Zeitraums (erst seit 2006) ihres Erscheinens mit Blick auf die angewandte Methodik und den gewählten Untersuchungszeitraum aus.

Die zu erhebenden Zeitschriften lassen sich auf der Grundlage ihrer (heutigen) Autor*innenrichtlinien und der tatsächlichen Veröffentlichungspraxis grob klassifizieren. Die PLANERIN, die RaumPlanung und die RAUM sind primär praxisorientiert, der Artikelumfang liegt zwischen 5.000 und 30.000 Zeichen (inkl. Leerzeichen) ${ }^{1}$. Die IzR wird von dem Bundesinstitut für Bau-, Stadt- und

\footnotetext{
${ }^{1}$ Angesichts der Praxisorientierung der PLANERIN, der RaumPlanung und der RAUM wird die Wissenschaftlichkeit dieser drei Zeitschriften seitens der planungswissenschaftlichen Fachgemeinschaft mitunter in Frage gestellt. Allerdings ist gemäß einer Stichprobe festzustellen, dass zumindest in der PLANERIN und der RaumPlanung deutlich mehr als die Hälfte der Autor*innen einen wissenschaftlichen Hintergrund haben und dass deutlich mehr als die Hälfte der mit Autor*innenangaben versehenen Artikel ein Literaturverzeichnis aufweisen, beides in steigendem Maße seit 1995. Bei der RAUM sind es jeweils etwas weniger als die Hälfte. Nachweisbar stell(t)en alle drei Zeitschriften ein Austauschformat für die wissenschaftliche Erkenntnisproduktion dar, was das einzig entscheidende Kriterium für Wissenschaftlichkeit im Rahmen dieser Arbeit ist. Vereinfachend werden daher alle genannten Zeitschriften in dieser Arbeit als planungswissenschaftlich bezeichnet. Auf die
} 
Raumforschung (BBSR) herausgegeben und ist dementsprechend an der Schnittstelle zwischen Wissenschaft, Politik und Praxis angesiedelt, allerdings mit einer primär wissenschaftlichen Autorenschaft (20.000 bis 55.000 Zeichen). Die disP und die RuR sind primär wissenschaftlich und vergleichsweise international ausgerichtet. Ihre Artikel werden vor ihrer Veröffentlichung einem double-blind peer review-Verfahren unterzogen (30.000 bis 75.000 Zeichen). Die ARL-FB stellen in erster Linie ein Veröffentlichungsformat für die jeweiligen thematisch begrenzten, maximal drei Jahre operierenden ARL-Arbeitskreise dar. Sie nehmen dementsprechend bezüglich der über die unterschiedlichen Ausgaben hinweg verteilten Gleichmäßigkeit des Inhaltes sowie der jährlichen Anzahl von Ausgaben und Artikeln eine Sonderposition ein. Das Format der ARL-FB changiert zwischen Monografie mit multipler Autorenschaft und Sammelband. Die Arbeitskreise sind in der Regel mit Akteur*innen sowohl der Wissenschaft als auch der Praxis besetzt. Mit Ausnahme der RAUM (Österreich) und der disP (Schweiz) werden alle Zeitschriften in Deutschland verlegt ${ }^{2}$.

Bei der Auswertung zu beachten sind die (unterschiedlichen) Zeiträume, die zwischen der Idee für einen Fachbeitrag, dem Beginn des Forschungsprozesses, der erstmaligen Einreichung des Artikels, der finalen Veröffentlichung sowie der Rezipierung durch die Fachöffentlichkeit liegen. Zunächst ist dabei grundsätzlich festzustellen, dass eine erhöhte Aufmerksamkeit gegenüber dem Gegenstand des Artikels seitens der Autor*innen nicht unbedingt nur im Jahr der Veröffentlichung anzunehmen ist, sondern auch in den unmittelbar vorangegangen Jahren. Darüber hinaus sind Unterschiede zwischen den ausgewählten planungswissenschaftlichen Zeitschriften zu berücksichtigen. So können einige Zeitschriften (insbesondere PLANERIN, RaumPlanung, RAUM) aufgrund redaktioneller Themensetzungen und weniger aufwändiger Artikelformate innerhalb weniger Monate auf veränderte Relevanzkriterien reagieren. Andere Zeitschriftenartikel sind dagegen das Produkt von teilweise mehrjährigen Forschungs- und review-Prozessen. Die ARLFB sind zudem das Resultat mehrjähriger, institutionalisierter Diskussionen. Von der formalen Initiierung eines Arbeitskreises bis zur Veröffentlichung des dazugehörigen Forschungsberichts vergehen in der Regel zwischen drei und fünf Jahren.

Unterschiede zwischen den jeweiligen Messwerten der unterschiedlichen Zeitschriften wird in den Auswertungen umso deutlicher hingewiesen.

${ }^{2}$ Auch die schweizerische disP und die österreichische RAUM stell(t)en im Zuge der untersuchten Themenkarrieren sowie generell nachweislich eine Kommunikationsplattform für deutsche Planungswissenschaftler*innen dar. Auf partiell auftretende Besonderheiten wird im Zuge der Auswertungen hingewiesen. 
Dank der freundlichen Unterstützung seitens der Verlage konnte der Arbeitsaufwand für die computergestützte Erhebung der Metadaten sowie des Volltextes insbesondere bei den jüngeren Jahrgängen gering gehalten werden. Der Volltext wurde digitalisiert und für die Auswertung aufbereitet. Die Metadaten wurden bereinigt und mithilfe der Microsoft-Office-Anwendung Access in eine relationale Datenbank eingespeist. In allen einschlägigen Artikeln aller Zeitschriften sowie zusätzlich in allen nicht-einschlägigen Artikeln der RuR (1995-2014) wurden die dort getätigten Zitationen erhoben (siehe Tab. 6.1). Die Auswahl der RuR für eine über die einschlägigen Artikel hinausgehende Zitationsanalyse ist aus forschungspragmatischen Erwägungen (hochwertige Digitalisierung im gesamten Untersuchungszeitraum und auswertungsfreundliche Formatierung der Literaturverzeichnisse) sowie aufgrund der Ausrichtung der Zeitschrift (Fokus auf Planungswissenschaft und vergleichsweise hohe Bedeutung des Zitats) erfolgt. Durch die Vollerhebung der RuR können die beiden Themenkarrieren vor dem Hintergrund eines planungswissenschaftlichen Gesamtdiskurses betrachtet werden, sodass die Besonderheiten der Themenkarrieren in jeder Phase sichtbar gemacht werden können (siehe bspw. Abb. 7.6 und Abb. 8.8).

Tab.6.1 Erhobene Daten strukturiert nach Zeitschrift und Datentyp

\begin{tabular}{|l|l|l|l|l|l|l|l|}
\hline & \multicolumn{2}{|l|}{ Vollerhebung (aller Artikel) } & \multicolumn{2}{l|}{ Einzelfallerhebung (nur einschlägiger Artikel) } \\
\hline & Metadaten & Zitationen & Volltext & Prüfung & Metadaten & Zitationen & Volltext \\
\hline PLANERIN & $1995-2014$ & - & - & $1995-2014$ & $1995-2014$ & $1995-2014$ & - \\
\hline RaumPlanung & $1995-2014$ & - & - & $1995-2014$ & $1995-2014$ & $1995-2014$ & - \\
\hline RAUM & $1995-2012$ & - & $1995-2012$ & $1995-2012$ & $1995-2012$ & $1995-2012$ & $1995-2012$ \\
\hline IzR & $1995-2014$ & - & $1995-2014$ & $1995-2014$ & $1995-2014$ & $1995-2014$ & $1995-2014$ \\
\hline RuR & $1995-2014$ & $1995-2014$ & $1995-2014$ & $1995-2014$ & $1995-2014$ & $1995-2014$ & $1995-2014$ \\
\hline disP & $1995-2014$ & - & $1995-2014$ & $1995-2014$ & $1995-2014$ & $1995-2014$ & $1995-2014$ \\
\hline ARL-FB & $1995-2014$ & - & $1995-2014$ & $1995-2014$ & $1995-2014$ & $1995-2014$ & $1995-2014$ \\
\hline
\end{tabular}

Quelle: Eigene Darstellung

Jede Zitation wurde mit allen abgebildeten Informationen digitalisiert und zusätzlich mit einem Code aus Autor*innen, Jahr und Titel versehen (bspw. „Gravert, Andreas; Günzel, Marian; Wiechmann, Thorsten\#2019\#Wie entstehen Themen in der Planungswissenschaft?"). Dieser Code transportiert alle zuverlässig in den Verzeichnissen aufgeführten und systematisch verwertbaren Informationen und fungiert als eindeutige Erkennung, anhand derer identische Veröffentlichungen softwaregestützt identifiziert werden können. Die an die Primärerhebung anschließende Datenbereinigung und Vereinheitlichung musste verschiedene Fehlerquellen berücksichtigen. Hierzu zählen insbesondere die unterschiedliche Handhabung 
multipler Urheberschaften (vollständige Aufzählung vs. „, et al.“, institutionelle*r Herausgeber*in vs. Autor*innen), die unterschiedliche Handhabung des Veröffentlichungsjahres bei mehrfachen Auflagen sowie die unterschiedliche Handhabung der Reihenfolge von Titel und Untertitel.

Nach der Erhebung und Bereinigung der Zitationsdaten liegen zum einen die Metadaten der planungswissenschaftlichen Artikel der sieben oben genannten planungswissenschaftlichen Zeitschriften vor und zum anderen die in diesen Artikeln zitierten Publikationen. Die dadurch hinzugewonnenen Publikationen und Autor*innen sind nicht mehr auf einen vorab definierten Zeitschriftenkatalog und Untersuchungszeitraum festgelegt. Verzeichnete Publikationen können also nun auch deutlich vor 1995 und außerhalb der sieben genannten Zeitschriften veröffentlicht worden sein. An dieser Stelle soll eine im Zuge der Darstellung der Fallstudien wichtige Begriffspräzisierung getroffen werden:

- ,planungswissenschaftliche Artikel' bezeichnet stets nur die Artikel in den sieben erhobenen Zeitschriften 1995-2014.

- ,Publikationen“ oder, Veröffentlichungen ' bezeichnet demgegenüber alle erhobenen Publikationen (1776-2015), einschließlich der nur als Zitierung vorliegenden.

Für die Auswertung sind nun primär sechs Tabellen in der Datenbank von Interesse (siehe Abb. 6.2): Das Publikationsverzeichnis inklusive Jahr, Titel und Ausgabe (T1), das Personenverzeichnis (T2), und das Verzeichnis der Themen (T3) sowie die drei Verknüpfungstabellen Autorenschaften (Personen-(veröffentlichen)->Publikationen) (VT1), Zitate (Artikel-(zitieren)>Publikationen) (VT2) und Inhalte (Artikel-(handeln von)->Themen) (VT3).

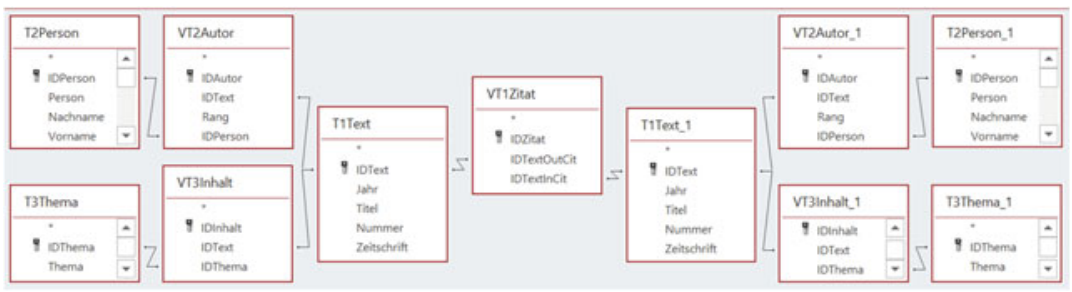

Abb.6.2 Struktur der relationalen Datenbank (Eigene Darstellung) 
Durch den relationalen Charakter der Datenbank werden diverse bibliometrische Abfragen und Analysen möglich, wie etwa eine thematisch einschlägige Zitationsanalyse (bspw. , welche Autor*innen werden in den Jahren 2005-2009 besonders häufig in solchen Artikeln zitiert, die Klimawandel zum Thema haben?').

Zusammen mit weiteren Verlagsdaten, wie etwa den Metadaten aller Ausgaben der ARL-FB vor 1995 (1.803 Artikel) sowie der RuR (1.973 Artikel), ergibt sich ein Datensatz mit 35.365 Veröffentlichungen, 16.881 Autor*innen und 25.850 Zitationen. Die Kennzahlen der erhobenen Artikel und der erhobenen Zitationen sind in Tab. 6.2 und Tab. 6.3 dargestellt.

Tab. 6.2 Erhobene Artikel nach Zeitschrift und Jahr

\begin{tabular}{l|l|l|l|l|l|l|l|c}
\hline Jahr & Gesamt & PLANERIN & RaumPlanung & RAUM & IzR & RuR & disP & ARL-FB \\
\hline 1995 & $\mathbf{3 1 2}$ & 48 & 36 & 60 & 70 & 55 & 36 & 7 \\
\hline 1996 & $\mathbf{3 3 0}$ & 65 & 44 & 58 & 57 & 44 & 30 & 32 \\
\hline 1997 & $\mathbf{3 3 0}$ & 65 & 44 & 56 & 70 & 41 & 36 & 18 \\
\hline 1998 & $\mathbf{3 1 7}$ & 56 & 33 & 50 & 68 & 40 & 30 & 40 \\
\hline 1999 & $\mathbf{3 8 6}$ & 92 & 34 & 55 & 75 & 44 & 36 & 50 \\
\hline 2000 & $\mathbf{3 9 4}$ & 112 & 43 & 52 & 72 & 47 & 36 & 32 \\
\hline 2001 & $\mathbf{3 6 4}$ & 88 & 41 & 48 & 80 & 45 & 30 & 32 \\
\hline 2002 & $\mathbf{3 8 4}$ & 78 & 43 & 56 & 77 & 34 & 41 & 55 \\
\hline 2003 & $\mathbf{3 5 7}$ & 94 & 34 & 53 & 73 & 37 & 38 & 28 \\
\hline 2004 & $\mathbf{3 1 6}$ & 86 & 36 & 52 & 59 & 34 & 37 & 12 \\
\hline 2005 & $\mathbf{3 9 7}$ & 92 & 35 & 49 & 80 & 38 & 41 & 62 \\
\hline 2006 & $\mathbf{3 8 3}$ & 110 & 35 & 47 & 67 & 42 & 28 & 54 \\
\hline 2007 & $\mathbf{3 4 8}$ & 109 & 39 & 40 & 70 & 40 & 29 & 21 \\
\hline 2008 & $\mathbf{3 8 2}$ & 108 & 35 & 49 & 124 & 39 & 27 & 0 \\
\hline 2009 & $\mathbf{3 4 3}$ & 99 & 36 & 48 & 74 & 30 & 30 & 26 \\
\hline 2010 & $\mathbf{3 5 4}$ & 100 & 37 & 49 & 80 & 34 & 45 & 9 \\
\hline 2011 & $\mathbf{3 5 6}$ & 126 & 31 & 47 & 67 & 28 & 32 & 25 \\
\hline 2012 & $\mathbf{3 8 8}$ & 116 & 54 & 40 & 56 & 36 & 33 & 53 \\
\hline 2013 & $\mathbf{3 0 3}$ & 121 & 64 & 0 & 49 & 29 & 33 & 7 \\
\hline 2014 & $\mathbf{3 0 9}$ & 102 & 49 & 0 & 53 & 30 & 51 & 24 \\
\hline Gesamt & $\mathbf{7 . 0 5 3}$ & $\mathbf{1 . 8 6 7}$ & $\mathbf{8 0 3}$ & $\mathbf{9 0 9}$ & $\mathbf{1 . 4 2 1}$ & $\mathbf{7 6 7}$ & $\mathbf{6 9 9}$ & $\mathbf{5 8 7}$ \\
\hline 0410 & & & & & & \\
\hline
\end{tabular}

Quelle: Eigene Darstellung 
Bei der Auswertung ist zu beachten, dass Zitationen, mit wenigen Ausnahmen, nur auf bereits veröffentlichte Beiträge entfallen. Theoretisch könnte deshalb eine Publikation aus dem Jahr 1994 1.400 Mal zitiert worden sein (von jedem der 1.400 mit Zitationen erhobenen Artikel einmal), während eine Publikation aus dem Jahr 2014 nur maximal 73 Mal zitiert worden sein kann (von jedem der 73 mit Zitationen erhobenen Artikel aus dem Jahr 2014 einmal), wobei tatsächlich nur 3,9 Prozent aller Zitationen auf dasselbe Jahr entfallen. Allerdings nimmt die Anzahl der Zitationen pro Artikel im Untersuchungszeitraum zu, im Falle der RuR bspw. um das Doppelte. Auch ist zu beachten, dass insbesondere Publikationen aus den jeweils vorangegangen vier Jahren zitiert werden, sodass etwa die meisten Zitationen aus der RuR nicht auf das Jahr 1994 entfallen, sondern auf das Jahr 2006. Grundsätzlich sind die quantitativen Ergebnisse aufgrund der teilweise geringen Fallzahlen sowie des begrenzt repräsentativen Publikationssegments der genannten Zeitschriften mit qualitativ erhobenen Informationen zu überprüfen und zu unterlegen. Werden quantitative Auswertungen zur Argumentation in den empirischen Kapiteln angeführt, so werden die Fallzahlen und die Berechnung offengelegt.

\subsubsection{Auswertung der quantitativen Daten: Formale Netzwerkanalyse}

Zur Auswertung der quantitativen Daten kommt neben der deskriptiven Statistik ${ }^{3}$ auch eine formale Netzwerkanalyse zum Einsatz. Die Netzwerkanalyse stellt eine Methode zur Erhebung und Auswertung relationaler Daten dar (Steinbrink et al. 2013: 15). In Abgrenzung zur qualitativen Netzwerkanalyse, arbeitet die formale Netzwerkanalyse mit quantitativen Daten und basiert auf der Graphentheorie. Sie nimmt also Graphen in den Blick, das heißt jeweils endliche Mengen an Knoten, die durch Kanten miteinander verbunden sind. In der Terminologie der sozialen Netzwerkanalyse werden Graphen als Netzwerke, Knoten als Akteur*innen und Kanten als Relationen oder Beziehungen bezeichnet (Heinze 2012: 192). Die Bedeutung eben jener Relationen für die untersuchten (sozialen) Ordnungen wird vor dem Hintergrund des ,Netzwerkparadigmas ' betont. Das heißt, nicht die individuellen Attribute und Eigenschaften einzelner Knoten (bspw. Akteur*innen)

\footnotetext{
${ }^{3}$ Die Bekanntheit der in der Empirie zum Tragen kommenden Auswertungsparameter und -methoden der deskriptiven Statistik wird vorausgesetzt.
} 


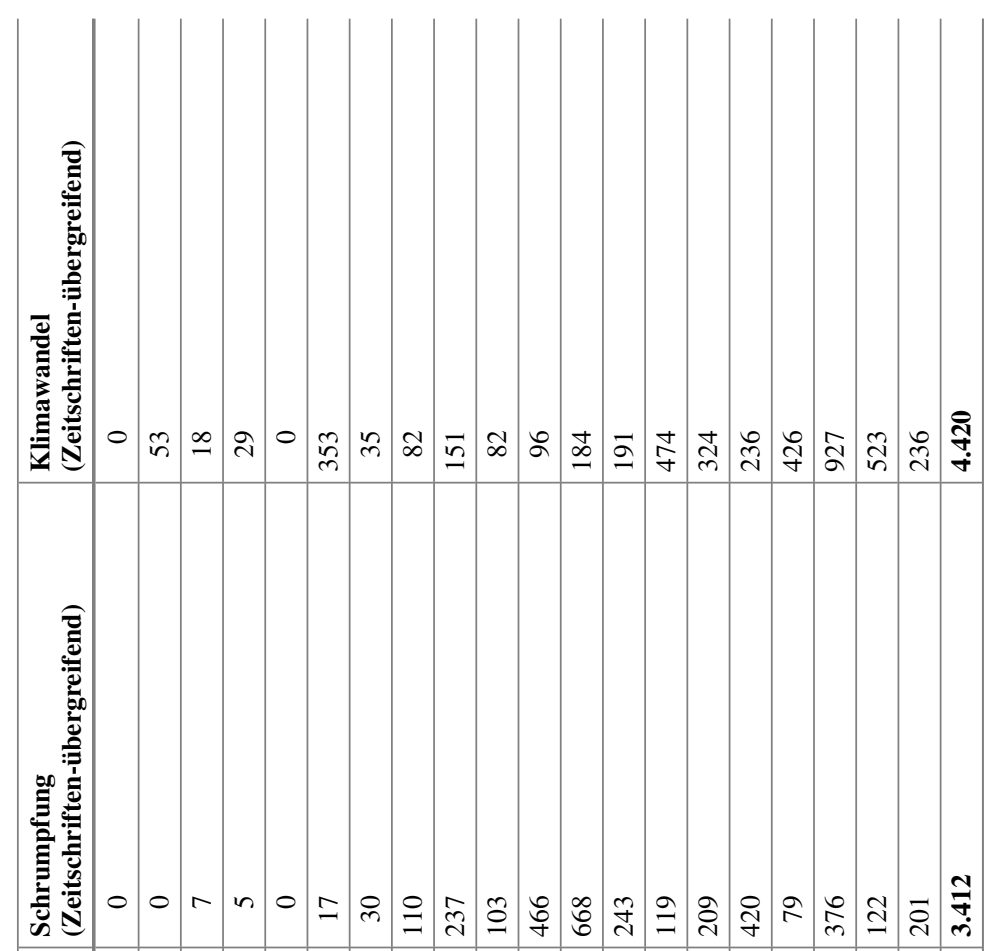

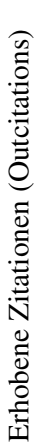

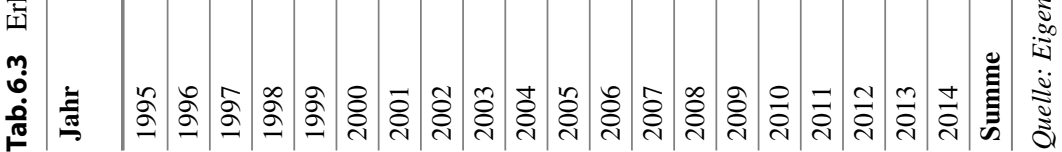


werden für die Beschreibung und Erklärung von (sozialen) Strukturen herangezogen, sondern die Beziehungen und Interdependenzen zwischen ihnen (Steinbrink et al. 2015: 435-439).

Die sozialwissenschaftliche Netzwerkforschung fokussiert auf die Untersuchung von sozialen Netzwerkstrukturen sowie auf die Einbettung von Akteur*innen in diese Strukturen. Auf der einen Seite wirken die Netzwerke von Akteur*innen als strukturelles Moment auf ihr soziales Verhalten ein. Das heißt, ihre Handlungen sind stets in soziale Beziehungen eingebettet (Herz et al. 2015b: o.S.; Granovetter 1985). Auf der anderen Seite sind die Strukturen des Netzwerks das Produkt dieser sozialen Handlungen der eingebetteten Akteur*innen. Soziale Netzwerke werden daher oft als eine Mesoebene betrachtet, als ,missing link" zwischen den Individuen (Mikroebene) und den gesellschaftlichen Strukturen (Makroebene) (Steinbrink et al. 2015: 435). In diesem Sinne wird die soziale Netzwerkanalyse als empirisches Programm anschlussfähig an die institutionalistisch orientierte Forschungsheuristik dieser Arbeit. Soziale Netzwerke ordnen den Akteur*innen Handlungsressourcen bzw. Sozialkapital zu und vermitteln Normen und Handlungsorientierungen, sie sanktionieren oder belohnen Handlungen, sie stellen Infrastrukturen für Transaktionen zwischen Akteur*innen bereit und sie strukturieren kollektive Akteur*innen (Diaz-Bone und Rainer 2006: 5-6; Scharpf 2006: 233-239).

Die quantitative Erhebung der Publikationsdaten ermöglicht die erstmalige formale Netzwerkanalyse der relationalen Strukturen der planungswissenschaftlichen Fachgemeinschaft. Planungswissenschaftler*innen können nun auf der Grundlage von Ko-Autorenschaften und Zitationen bspw. bezüglich ihrer Position im Netzwerk analysiert werden, die auf der einen Seite ihnen selbst Voroder Nachteile im sozialen Tausch (Sozialkapital) verschafft und die auf der anderen Seite eine Funktion für die Kommunikations- oder Organisationsfähigkeit des Netzwerks einnimmt. Auch die Identifizierung unverbundener Netzwerksegmente (struktureller Löcher) bzw. die Frage, welche Akteur*innen wann die einzelnen Netzwerksegmente durch neue Beziehungen verbinden, kann aufschlussreich für die Analyse von Themenkarrieren und darüber hinaus sein.

\section{Methodische Grundlagen der Netzwerkanalyse}

Ausgangspunkt jeder Netzwerkanalyse ist die Definition der Knoten und der Beziehungen sowie die Abgrenzung des Netzwerks. Die Abgrenzung des Netzwerks variiert in dieser Arbeit von Auswertung zu Auswertung (bspw. , alle einschlägigen Artikel 1995-2014'). Als Knoten werden, je nach spezifischer Fragestellung, einerseits Publikationen (etwa ,welche Publikationen sind ähnlich?') und andererseits Autor*innen (etwa ,welche Autor*innen sind zentral?') zum Gegenstand formaler Netzwerkanalysen gemacht. Als Beziehungen werden in 
dieser Arbeit zwei Arten erhoben: Ko-Autorenschaften und Zitationen. Aus den Ko-Autorenschaften ergibt sich ein Ko-Publikationsnetzwerk. Dadurch, dass alle erhobenen Publikationen mitsamt Autor*innen vorliegen, lassen sich nicht nur aus den planungswissenschaftlichen Artikeln, sondern auch aus den darin referenzierten Publikationen Ko-Autorenschaften auswerten. Beispielsweise lassen sich jeweils Ko-Publikationsnetzwerke zu den beiden Themen Schrumpfende Städte und Klimawandel analysieren und die Entwicklung der Graphen im Zeitverlauf anhand des Erscheinungsjahrs der Publikationen betrachten. Zitationen bieten sich als Indikator für Wissensnetzwerke an. Zitationsnetzwerke lassen sich zeitschriftenübergreifend bezüglich der beiden Themenkarrieren, anhand der RuR-Zitationen aber auch themenunabhängig auswerten. Beispielsweise lässt sich betrachten, welche Autor*innen zu welcher Zeit besonders häufig in einschlägigen Artikeln zitiert werden und inwiefern die Fokussierung auf diese Autor*innen auch themenübergreifend $\mathrm{zu}$ beobachten ist.

Die Netzwerkanalyse unterscheidet weiter drei grundlegende Eigenschaftsaspekte: (1.) Eigenschaften der Knoten (bspw. Zentralität), (2.) Eigenschaften der Kanten (bspw. Gewicht bzw. Intensität der Beziehung) sowie (3.) Eigenschaften der Netzwerkstruktur (bspw. Dichte) (Diaz-Bone und Rainer 2006: 7). Darüber hinaus enthalten soziale Netzwerke Attributinformationen, die die einzelnen Knoten und Kanten charakterisieren (bspw. Namen der Akteur*innen, Art der Beziehung) (Holzer 2015: 34).

Die methodischen Verfahren, die in der Netzwerkanalyse Anwendung finden, lassen sich in zwei unterschiedliche Zugänge differenzieren: Verfahren, die auf Parameter der Kohäsion fokussieren (relationale Analysen), und Verfahren, die die Äquivalenz (positionale Analysen, Blockmodellanalyse) in den Blick nehmen (Herz et al. 2015a). Zu Parametern der Kohäsion lassen sich Berechnungen bezüglich Beziehungsdichte, Cluster-Koeffizienten oder der Kohäsion zählen (siehe Tab. 6.4) (Herz et al. 2015a; Heinze 2012: 195). Demgegenüber nimmt die Äquivalenz die Ähnlichkeit von Akteur*innen auf der Grundlage ihrer Beziehungsstrukturen in den Blick. Strukturell äquivalent sind zwei Akteur*innen dann, wenn sie identische Beziehungen zu allen anderen Akteur*innen des Netzwerks haben. Regulär äquivalent sind sie, wenn sie gleichartige Beziehungen zu wiederum regulär ähnlichen (aber nicht zwangsweise denselben) Akteur*innen haben (Heidler 2006: 10-11). Die Äquivalenz mehrerer Akteur*innen im Netzwerk erlaubt Rückschlüsse auf gleichartige Rollen, sodass bestimmte Akteur*innengruppen unabhängig von direkten Beziehungen zueinander gebildet und analysiert werden können (Herz et al. 2015a).

Die netzwerkanalytischen Berechnungen und Darstellungen werden mit dem Softwarepaket Gephi erstellt. In den Grafiken kommen die Layout-Algorithmen Force Atlas sowie Force Atlas 2 (Jacomy 2009) zum Einsatz. Force-Algorithmen 
versehen klassischerweise alle Knoten mit einer Abstoßungskraft, während gleichzeitig die Kanten eine zusammenziehende Wirkung ausüben. Knoten liegen umso näher beieinander je häufiger (Anzahl der Kanten) und stärker (Gewicht der Kanten) sie miteinander verbunden sind und je ähnlicher sie sich in Bezug auf ihre Beziehungen zu dritten Knoten sind. Darüber hinaus schiebt Force Atlas die Knoten mit den meisten Kanten (hubs) tendenziell auseinander, während die mit ihnen direkt verbundenen Knoten in Clustern um die hubs herum ausgerichtet sind. So wird erreicht, dass sich so wenige Kanten wie möglich gegenseitig kreuzen (Paranyushkin 2011: 10). Der ebenfalls angewandte Noverlap Layout-Algorithmus dient dazu, Knoten bei Überlappungen zugunsten der besseren Lesbarkeit (minimal) auseinander zu schieben.

\section{Grundbegriffe der Bibliometrie und der Netzwerkanalyse}

Im empirischen Teil dieser Arbeit (Kp. 7 und 8) werden einige Begriffe aus der Netzwerktheorie sowie der Bibliometrie verwendet, die im Format eines Glossars im Folgenden (Tab. 6.4) kurz erläutert werden.

Tab.6.4 Grundbegriffe der Bibliometrie und der Netzwerkanalyse

Ein $\underline{\operatorname{Graph}}(\mathrm{G}=(\mathrm{N}, \mathrm{L}))$ besteht aus zwei endlichen Mengen: den ,Knoten ' (nodes) des Graphen $(\mathrm{N}(\mathrm{G})$ ) und Kanten (links) des Graphen $(\mathrm{L}(\mathrm{G})$ ), die die Knoten verbinden. Ein dynamischer Graph betrachtet die Entwicklung des Netzwerks im Zeitverlauf.

Ein $\underline{P f a d}$ ist eine Verbindung zwischen zwei Knoten, die ein oder mehrere Kanten, jede Kante jedoch maximal einmal, miteinbeziehen kann. Im Rahmen dieser Arbeit wird nur der Kürzeste Pfad betrachtet, also jener mit der kleinsten Kantenzahl.

Eine Kante kann mit einem Gewicht versehen sein, wenn Unterschiede bezüglich der Intensität von Kanten bzw. Beziehungen vorliegen. Eine stark gewichtete Beziehung verursacht mehr Anziehung bzw. weniger Widerstand als eine schwache.

In einem ungerichteten Graphen, beruht jede Beziehung zwischen zwei Knoten auf Gegenseitigkeit. Indem sich Ko-Autorenschaften aus gemeinsam verantworteten Publikationen ergeben, sind sie ungerichtet (bspw. Ko-Autor*in1<->Ko-Autor*in2).

In einem gerichteten Graphen hat jede Beziehung eine Richtung, die Kanten werden daher als $\underline{\text { Pfeile }}$ bezeichnet. Indem Zitationen sich aus dem einseitigen Verweis einer Publikation auf eine andere ergeben, handelt es sich um gerichtete Beziehungen (bspw.

Zitierende*r->Zitierte*r).

Adjazent sind zwei Knoten, wenn sie durch eine Kante direkt miteinander verbunden sind. In einem gerichteten Graphen ist der Knoten A adjazent $z u$ Knoten B, wenn die beiden Knoten durch den Pfeil (A->B) verbunden sind. Der Knoten A ist adjazent von Knoten B, wenn die beiden Knoten durch den Pfeil (B->A) verbunden sind. 
Tab.6.4 (Fortsetzung)

Ein soziales Netzwerk kann als Graph betrachtet werden, wobei die Akteur*innen durch Knoten repräsentiert sind und die Beziehungen zwischen den Akteur*innen durch Kanten.

Ko-Publikationsnetzwerke bestehen aus (gegenseitigen) Ko-Autorenschaften und stellen somit ungerichtete Graphen dar.

Zitationsnetzwerke bestehen immer aus gerichteten Relationen (bspw. A zitiert B).

Outcitations (von Knoten A) sind in einem Zitationsnetzwerk ausgehende Pfeile (A->), während Incitations (von Knoten B) dessen eingehende Pfeile darstellen ( $->\mathrm{B})$. Analog sind die Outcitations eines Jahres die von den Artikeln dieses Zeitraums ausgehenden Zitierungen älterer Publikationen. Die Incitations eines Jahres sind dagegen die auf die Publikationen dieses Zeitraums eingehenden Zitierungen jüngerer Artikel.

Zitiert ein Knoten sich selbst (A->A), spricht man von einer Selbstzitation.

Der Grad eines Knotens bezeichnet die Anzahl seiner Kanten. In einem gerichteten Netzwerk (bspw. Zitationsnetzwerk) bezeichnet der Eingangsgrad die Anzahl der eingehenden (Incitations), der Ausgangsgrad die Anzahl der ausgehenden Verbindungen (Outcitations).

Ein*e Akteur*in in einem sozialen Netzwerk ist zentral, wenn er*sie eine wichtige Stellung in dem sozialen Netzwerk einnimmt. Diese Wichtigkeit lässt sich differenzieren anhand der Gradzentralität, die sich auf Menge und/oder Gewicht der direkten Beziehungen eines Knotens bezieht. Daneben beschreibt die closeness-Zentralität die Nähe zu allen anderen Akteur*innen, das heißt die durchschnittliche Länge der Pfade zu allen anderen Akteur*innen. Die betweenness-Zentralität bezieht sich auf die strategisch günstige Lage. Sie manifestiert sich in der Anzahl kürzester Pfade (zwischen beliebigen Knotenpaaren), die durch einen Knoten führen, was wiederum auf eine Maklerfunktion zwischen bestimmten Bereichen des Netzwerks hinweisen kann. Eigenvektor-Zentralität oder auch Status ist ein rekursives Konzept, bei dem Akteur*innen umso zentralere Positionen einnehmen, je zentraler die Akteur*innen sind, mit denen sie Beziehungen unterhalten.

Autoritäten sind in einem gerichteten Graphen Knoten mit hohem Eingangsgrad, sie verfügen über ein hohes Prestige. Rang-Prestige ist analog zur Eigenvektor-Zentralität ein rekursives Konzept von Prestige, bei dem derjenige über ein hohes Rang-Prestige verfügt, der Pfeile von anderen Akteur*innen unterhält, die selbst ein hohes Prestige besitzen. Demgegenüber stellen hubs Knoten mit hohem Ausgangsgrad dar, sie verfügen über eine hohe Aktivität.

Fehlende Beziehungen zwischen Akteur*innen oder Teilgruppen werden als Strukturelle Löcher bezeichnet. Ein*e Makler*in, der*die zwischen verschiedenen Akteur*innen oder Teilgruppen Beziehungen unterhält, die ihrerseits nicht verbunden sind, hat die Möglichkeit zwischen den Kontakten zu vermitteln und Informations- und Kontrollvorteile zu realisieren. Eine Form der Maklerposition ist der*die Gatekeeper*in, der*die die eigene Gruppe mit dem übrigen Netzwerk bzw. mit einer anderen Teilgruppe verbindet und kontrolliert, welche Informationen in die eigene Teilgruppe hinein bzw. aus dieser hinaus gelangen.

(Fortsetzung) 
Tab.6.4 (Fortsetzung)

Die Dichte eines Graphen ist definiert als die Anzahl der Kanten (bzw. Pfeile) geteilt durch die Anzahl aller möglichen ungeordneten Paare unterschiedlicher Knoten. Diese Anzahl beträgt in ungerichteten Graphen mit $\mathrm{g}$ Knoten $\mathrm{g} \cdot(\mathrm{g}-1) / 2$ und in gerichteten Graphen mit $\mathrm{g}$ Knoten $\mathrm{g} \cdot(\mathrm{g}-1)$. In einem vollständigem Graph ist jeder Knoten mit jedem anderen Knoten verbunden und seine Dichte entspricht 1.

Ein Graph teilt sich häufig in nicht miteinander verknüpfte Komponenten, wobei die Hauptkomponente die größte Zahl wenigstens indirekt miteinander verbundener Knoten aufweist. Bei zunehmender Integration des Graphen bildet sich in der Regel eine Gigantische Komponente, in der ein Großteil der Knoten wenigstens indirekt miteinander verbunden sind, sodass davon unabhängige Subkomponenten nur noch sehr viel kleiner als die Gigantische Komponente ausfallen.

Eine Menge von Akteur*innen, die, verglichen mit dem übrigen Netzwerk, eine besonders hohe Verbundenheit untereinander aufweist, nennt sich Teilgruppe oder Cluster. Bei maximaler Verbundenheit (jeder mit jedem) spricht man von einer $C$ Clique. Akteur*innen, die keinerlei Verbindung im Netzwerk haben, werden als isolates bezeichnet.

Knoten werden in Modularitätsklassen zusammengefasst, wenn sie intern stärker miteinander verbunden sind als mit dem übrigen Netzwerk bzw. mit den Knoten anderer Modularitätsklassen.

Quelle: Eigene Darstellung basierend insbesondere auf Schnerch 2019; Trappmann et al. 2011: 51-61; Steinbrink et al. 2010; Mutschke 2010; Leifeld und Malang 2009; Jansen 2003

\subsection{Qualitative Analyse: Leitfadengestützte Interviews}

Das Ziel der qualitativen Empirie ist es, die Strukturen und individuellen Handlungsbedingungen der Planungswissenschaftler*innen offenzulegen, die für die Entwicklung der beiden Themen Schrumpfende Städte und Klimawandel ausschlaggebend waren. Im Zuge der angestrebten Mikrofundierung des Makrophänomens wird die Forschungsfrage nunmehr auf die empirisch zu untersuchende Akteur*innenebene heruntergebrochen. Sie lautet ,Wie wählen Planungswissenschaftler*innen ein Thema und warum?‘ Es wird also im Sinne der Forschungsheuristik nach den jeweils relevanten Handlungsbedingungen der Planungswissenschaftler*innen im Kontext ihrer Entscheidungen zugunsten bzw. zulasten eines Themas gefragt. Dem institutionalistischen Programm folgend wird die Veränderung dieser Handlungsbedingungen als Erklärungsmoment für Themenkarrieren anvisiert.

Für die Rekonstruktion der Entscheidungssituationen von Planungswissenschaftler*innen lassen sich einige Anhaltspunkte durch die Auswertung ihrer Publikationen sowie ihrer öffentlich zugänglichen (forschungs-)biografischen Daten identifizieren. Darüber hinaus werden aber Informationen benötigt, über die nur diese Personen selbst verfügen - etwa in Bezug auf nicht wahrgenommene Handlungsoptionen, persönliche Motive oder Wahrnehmungen struktureller 
Anreizstrukturen. Um dieses Wissen schöpfen zu können, ist das leitfadengestützte Interview mit einem Fokus auf die retrospektive Analyse der maßgeblichen forschungsbiografischen Entscheidungssituationen in Bezug auf die Themenwahl bzw. die Änderung des Forschungsinhaltes die Methode der Wahl.

Die Vorbereitung, Durchführung und Auswertung der Methode leitfadengestützter Interviews im Rahmen dieser Arbeit erfordert eine hohe Sensibilität gegenüber spezifischen Antworttendenzen, auf die in Abschnitt 6.4.1 eingegangen wird. Um diesen zu begegnen, werden die Interviews durch die Erhebung quantitativer, textbasierter sowie biografischer Informationen vorbereitet. Hierbei werden die persönlichen Forschungsbiografien und diachronen Wissensstrukturen der Befragten auf der Grundlage von Publikations- und web-basierten Daten analysiert, visualisiert und daraus Detailfragen abgeleitet (Kp. 6.4.2). Ebenfalls zur Vorbereitung der Interviews und zur Entwicklung der Leitfaden werden aus der Theorie abgeleitete Aspekte, die für die Beantwortung der Forschungsfrage ein hohes Erklärungspotenzial besitzen, in Form eines Fragenkatalogs operationalisiert und systematisiert (Kp. 6.4.3). Es folgt die Auswahl der Interviewpartner*innen (Kp. 6.4.4) und die Durchführung und Auswertung der Interviews (Kp. 6.4.5).

\subsubsection{Funktion und Herausforderungen leitfadengestützter Interviews}

Zur Beantwortung der Frage, wie und warum Wissenschaftler*innen ein Thema aufgreifen und es weiterverfolgen, gilt es, die jeweiligen Entscheidungssituationen $\mathrm{zu}$ rekonstruieren, das heißt, die Handlungsbedingungen zu identifizieren, die für die Themenentscheidungen ausschlaggebend waren. Hierfür wird Wissen benötigt, über welches exklusiv die jeweils entscheidenden Personen verfügen und welches durch die Methode des leitfadengestützten Interviews erhoben werden kann.

Das leitfadengestützte Interview ist ein nicht-standardisiertes Interview, bei dem der*die Interviewer*in eine Liste offener Fragen (den Leitfaden) zur Grundlage des Gesprächs macht. Es stellt damit einen Kompromiss aus narrativen und standardisierten Interviewformen dar, der sowohl Offenheit als auch Vergleichbarkeit gewährleisten soll. Nach Gläser und Laudel (2006: 107) wird der Leitfaden eingesetzt, wenn „in einem Interview mehrere unterschiedliche Themen behandelt werden müssen, die durch das Ziel der Untersuchung und nicht durch die Antworten des Interviewpartners bestimmt werden“ und wenn gleichzeitig ,auch einzelne genau bestimmbare Informationen erhoben werden müssen ". Die Orientierung an einem Leitfaden soll die Zielführung des Interviews sicherstellen, ermöglicht aber gleichzeitig eine offene Gesprächsführung, während derer die 
befragte Person die Möglichkeit hat, eigene Erklärungsansätze, Gesichtspunkte, Schwerpunktsetzungen und Kontextinformationen einzubringen. Der*Die Interviewer*in kann flexibel auf Äußerungen oder Reaktionen der befragten Person reagieren, ist aber in der Verantwortung, den Fokus des Gesprächs aufrecht zu erhalten. Eine besonders ausgeprägte Steuerungsfunktion kann die Leitfadenorientierung folglich im Hinblick auf den Ausschluss unergiebiger Themen ausüben (Flick 2006: 139-140).

Allerdings ergeben sich aus der Interdependenz zwischen interviewender und interviewter Person sowie aus der retrospektiven Betrachtung teilweise weit zurückreichender, flüchtiger Entscheidungen mehrere Punkte, die bei der Vorbereitung, Durchführung sowie in der Auswertung der Interviews eines besonderen Augenmerks bedürfen: Zunächst muss das Problem der sozialen Erwünschtheit in besonderem Maße berücksichtigt werden, weil Fragen nach persönlichen Handlungsorientierungen und -ressourcen als sensibel wahrgenommen werden können. Dies gilt insbesondere, weil die interviewte Person, der Interviewer sowie ein Großteil der Adressaten dieser Forschungsarbeit zu ein und derselben Fachgemeinschaft zu zählen sind. Sie beobachten sich also teilweise gegenseitig und teilen einander Handlungsressourcen, wie bspw. Reputation, zu. Den befragten Personen ist daher grundsätzlich eine verstärkte Tendenz $\mathrm{zu}$ unterstellen, ein positives Bild der eigenen Handlungen zeichnen zu wollen. Dabei variieren zum einen die subjektiven Kriterien sozialer Erwünschtheit gemäß ihrer präferierten Rollenverständnisse (bspw. legt die eine Person besonderen Wert darauf, wissenschaftliche Pionierarbeit geleistet zu haben, eine andere Person möchte ihre Handlungen auch als gesellschaftspolitischen Aktivismus verstanden wissen) (vgl. Lamker 2017). Zum anderen variiert der Grad der (durch die befragte Person wahrgenommenen) Interdependenz zum Interviewer zwischen nicht-vorhanden (bspw. wegen Ruhestands) und direkter Austauschbeziehung.

Mit der teilweise großen Zeitspanne zwischen dem Interview und den zu rekonstruierenden Entscheidungen sind weitere Erhebungsprobleme verbunden. Zunächst lässt das Erinnerungsvermögen mit zunehmendem zeitlichen Abstand nach, sodass es zu Vergessen und Fehl-Erinnern kommen kann. Hinzu kommt ein ,Nadel-im-Heuhaufen'-Problem, denn es gilt, bestimmte, teilweise flüchtige Entscheidungen in einer permanenten Veränderungen unterworfenen Forschungsbiografie zu identifizieren, die möglicherweise erst im Nachhinein und nur im Rahmen der hier vorliegenden Fragestellung als relevant eingestuft werden (Gläser und Laudel 2015a: 300). Des Weiteren stellt die retrospektive Rationalisierung von Handlungen ein Erhebungsproblem dar. Dies gilt insbesondere für das Feld der Wissenschaft, da hier Wissensansprüche besonders umstritten sind und der wissenschaftlich-rationalen Herleitung von Forschungsentscheidungen große Bedeutung beigemessen wird. 
Den genannten Antworttendenzen soll durch die Triangulation unterschiedlicher Datenquellen (siehe Kp. 6.4.2), die bezüglich der genannten Faktoren sensibilisierte Erstellung des Leitfadens (siehe Kp. 6.4.3), die auf objektiven Kriterien beruhende Auswahl der zu befragenden Personen (siehe Kp. 6.4.4), die aufmerksame und informierte Gesprächsführung sowie die nachträgliche Prüfung und achtsame Auswertung (siehe Kp. 6.4.5) begegnet werden. Dabei hat das Untersuchungsfeld der Wissenschaft den Vorteil, dass eine sehr große Menge weit zurückreichender und aufschlussreicher Daten systematisch erfasst und öffentlich zugänglich vorliegt. So sind in Publikationen nicht nur der Forschungsprozess und der Wissensstand zum Zeitpunkt der jeweiligen Veröffentlichung textlich konserviert. Anhand von Ko-Autorenschaften und Zitationen lassen sich auch persönliche Bekanntschaften und diachrone Wissensstrukturen nachvollziehen. Nicht zuletzt entfaltet die Zugehörigkeit des Forschers (bzw. Interviewers) und der erforschten Personen (bzw. der Interviewten) zu ein und derselben Fachgemeinschaft hier auch einen positiven Effekt, schließlich muss das benötigte Verständnis der Inhalte sowie der sozialen Mechanismen, mit denen die Interviewten befasst sind bzw. waren, nicht erst aufwändig erarbeitet werden.

\subsubsection{Erhebung und Auswertung von Forschungsbiografien}

Um die aggregierte Themenwahl retrospektiv nachvollziehen zu können, sind die Entscheidungssituationen der fachgemeinschaftlichen Akteur*innen zu rekonstruieren: Was hat wann dazu geführt, dass sich die Planungswissenschaftler*innen für oder gegen die Beschäftigung mit dem Thema entschieden haben? Für diese Frage werden die Erinnerungen und Bewertungen derjenigen Personen benötigt, die diese Entscheidungen getroffen haben. Um diese Informationen im Zuge der Interviews möglichst unverfälscht und tiefschürfend erfassen und wenn möglich validieren zu können, werden bibliometrische Daten und biografische Informationen über die Interviewten vorab recherchiert und ausgewertet. Die erhobenen Informationen werden - wo sinnvoll (siehe Kp. 6.4.5) - visualisiert, großformatig ausgedruckt und als Impuls sowie zur beiderseitigen Orientierung in den Gesprächssituationen eingesetzt.

Die Erstellung und Nutzung solcher visualisierter, bibliometrisch informierter Forschungsbiografien zur Unterstützung von Interviews wurde von Gläser und Laudel im Rahmen ihrer Forschungen zu Innovationen in der Wissenschaft entwickelt und getestet (Gläser und Laudel 2007, 2009, 2015a; Laudel und Gläser 2007). Dem Instrument der ,bibliometric research trails “ liegt die Betrachtungsweise zugrunde, dass sich wissenschaftliche Erkenntnisentstehung auf Ebene der 
Fachgemeinschaft, aber auch auf Ebene des Individuums als diachrone Wissensstruktur erfassen lässt. Diese besteht aus verschiedenen, sich überschneidenden Forschungspfaden, das heißt Sequenzen thematisch zusammenhängender Projekte, in denen frühere Forschungsprozesse als Input für spätere dienen (siehe Kp. 4.2.3). Aus dieser Perspektive folgt eine Betonung der Pfadabhängigkeit von Forschungsentscheidungen:

„The researcher's own research biography creates a unique combination of knowledge that has been accumulated in prior research, the frames guiding the perception and evaluation of new knowledge, and individual interests and preferences. "(Gläser 2012a: 7)

Die Forschungspfade lassen sich mit Hilfe bibliometrischer Daten und Methoden erheben. Diese haben den Vorteil, objektiv zu sein. ,Objektivität‘ heißt hier, dass die Analyse unabhängig von den $a d$ hoc-Interpretationen der eigenen Karriere seitens der Interviewten selbst erfolgt, was Verzerrungen durch die retrospektiven Rationalisierungen der Informant*innen vermeidet. Stattdessen kann anhand der analysierten Publikationen, ihrer Inhalte, Kooperationen und Zitationen ein weitgehend verzerrungsfreies Bild davon gezeichnet werden, auf welchem Wissensstand sich die Wissenschaftler*innen befanden, als sie ihre jeweiligen Erkenntnisse veröffentlichten. Durch diese Vorteile eignen sich bibliometrische Methoden besonders, um die qualitativen und subjektiven Interview-basierten Daten zu triangulieren (Gläser und Laudel 2015a: 303). Bibliometrisch informierte Visualisierungen von Forschungsbiografien, im Folgenden kurz ,research trails", werden deshalb bei der Vor- und Nachbereitung sowie während der Interviews eingesetzt.

Zur Erstellung eines research trail wird die Forschungsbiografie der interviewten Person erhoben, ausgewertet und im Zeitverlauf dargestellt. Verschiedene Informationen werden dafür aus der eigenen Datenbank sowie auf Basis einer Bibliotheks- und Internetrecherche gesammelt: beruflicher Werdegang, Publikationen, Zitationen, Forschungsschwerpunkte, Beteiligungen an Forschungsprojekten, Mitgliedschaften sowie Qualifizierungsarbeiten und Kooperationen. Die verschiedenen Daten werden mit einem Fokus auf thematische Verläufe und Brüche ausgewertet und - wo sinnvoll - visualisiert. Kern der Visualisierung ist die Rekonstruktion der Forschungspfade auf der Basis bibliometrischer Daten nach dem Prinzip einer diachronen Netzwerkabbildung (siehe Abb. 6.3 und Abb. 6.4). Diese rückt die Publikationen der Akteurin bzw. des Akteurs in den Fokus, die als farbliche Kreise dargestellt sind. Deren Größe variiert entsprechend der in Incitations manifestierten Bedeutung, die die Publikation im Rahmen der betrachteten Forschungsbiografie innehat. Hierfür werden auf Grundlage der eigenen 
planungswissenschaftlichen Datenbank (PW DB) sowie der Google ScholarDatenbank $^{4}$ (GS DB) jeweils Bedeutungskoeffizienten (BK) gebildet und addiert. Der so berechnete BK Gesamt ist Grundlage für die Berechnung der Kreisgröße.

BK Gesamt $=\frac{\text { Incitations der Publikation aus PW DB }}{\text { Alle Incitations der Person aus PW DB }}+\frac{\text { Incitations der Publikation aus GC DB }}{\text { Alle Incitations der Person aus GS DB }}$

Der BK Gesamt soll den deutschsprachigen, planungswissenschaftlichen und einschlägigen Fokus sowie die Nachvollziehbarkeit der eigenen Datenbank (bei gleichzeitig kleinen Fallzahlen) mit den großen Fallzahlen von Google Scholar (bei gleichzeitig disziplinübergreifender, nicht kontrollierbarer Datenerhebung mit einer vielfach höheren Gewichtung englischsprachiger Publikationen) kombinieren und die jeweiligen Schwächen ausgleichen (zur Verwendung von Google Scholar in bibliometrischen Analysen siehe Saßmannshausen 2012: insb. 151-152). Die eigene Datenbank trifft also reliable Aussagen über die Bedeutung bestimmter Publikationen im deutschsprachigen, planungswissenschaftlichen und einschlägigen Diskurs, während die Zitationsindizes von Google Scholar die Bedeutung bestimmter Publikationen für den individuellen, themenunabhängigen Reputationserwerb der Akteur*innen besser wiedergeben.

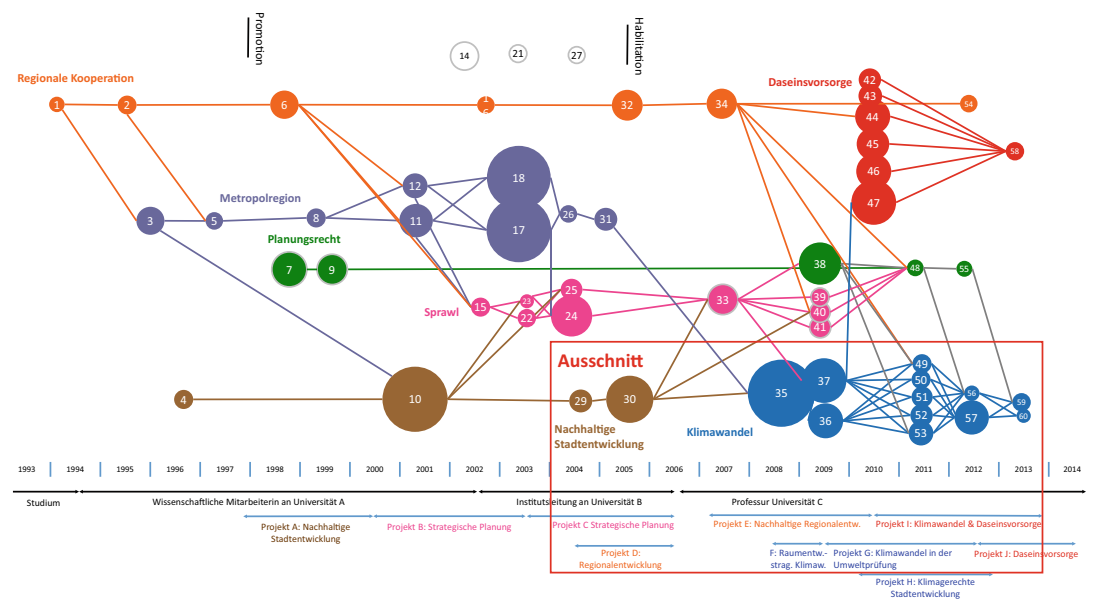

Abb.6.3 Beispielhafte Visualisierung einer Forschungsbiografie bzw. eines research trail mit dem vorübergehenden Themenschwerpunkt Klimawandel (Originalformat: DIN A0)

${ }^{4}$ Auf die Google Scholar-Daten wird mit Hilfe der Software Publish or Perish zugegriffen. 


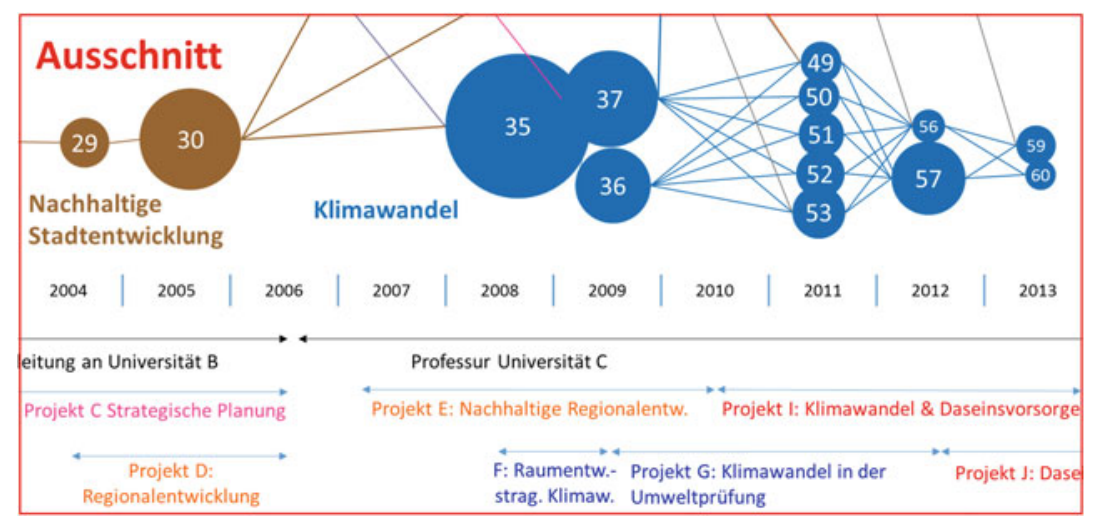

Abb.6.4 Ausschnitt der Thematisierung des Klimawandels im research trail (Eigene Darstellung basierend auf Gläser und Laudel (2007, 2009, 2015a))

Die Farbe der Kreise ergibt sich aus der Zuordnung der repräsentierten Publikation zu dem jeweils im Vordergrund stehenden Thema, die wiederum primär auf der Basis der Publikationstitel händisch erfolgte. Inhaltliche Verknüpfungen zwischen den Publikationen (Forschungspfade) sind darüber hinaus mit farbigen Verbindungslinien dargestellt, was insbesondere dann eine Zusatzinformation bereithält, wenn ein neuer Forschungspfad (bspw. Umweltgerechtigkeit) aus einem oder mehreren bestehenden Forschungspfaden (bspw. Umweltindikatoren, Umweltrecht) entsteht. Ebenfalls dargestellt werden Karrierestationen, Drittmittelprojekte und Ko-Autorenschaften. Zu jedem research trail gehört eine Legende, in der Erscheinungsjahr, Titel, Ko-Autor*innen und identifizierte Themen der einzelnen Publikationen verzeichnet sind.

\subsubsection{Erstellung der Leitfaden}

Die Formulierung und Zusammenstellung der Fragen für das Interview (der Leitfaden) erfolgt auf Basis (1.) der dieser Arbeit zugrundeliegenden Fragestellung sowie dem aus der Theorie abgeleiteten analytischen Rahmen, (2.) der individuellen Forschungsbiografie und der daraus abgeleiteten, potenziell aufschlussreichen Entscheidungssituationen sowie (3.) des Wissens um mögliche Antworttendenzen.

Der analytische Rahmen fungiert für die empirische Datenerhebung als eine Art Scheinwerfer, indem er die Aufmerksamkeit auf jene Aspekte richtet, denen ein besonders hohes Erklärungspotenzial beigemessen wird (siehe Kp. 5). Für die 
Interviewvorbereitung heißt das, dass die Faktoren und Mechanismen, die potenziell bei den Themenentscheidungen Einfluss genommen haben, im Leitfaden operationalisiert werden. Hierzu gehören akteur*innenbezogene Fragen (bzgl. der Handlungsorientierungen und -ressourcen einschließlich sozialer Beziehungen) sowie strukturbezogene Fragen (bzgl. strukturell vermittelter Handlungsspielräume und Erwartungshaltungen) in den jeweiligen Entscheidungssituationen.

Die forschungsbiografische Auswertung ermöglicht die individuelle Anpassung des aus der Heuristik abgeleiteten Fragenkatalogs auf die im Sinne der Fragestellungen jeweils besonders aufschlussreichen Entscheidungssituationen bzw. auf die ihnen jeweils zugrundeliegenden Handlungsbedingungen. Auf dieser Basis werden die Leitfaden erstellt, sodass diese neben Interview-übergreifenden Fragen (bspw. ,Wie schätzen Sie den weiteren Forschungsbedarf zum Thema ein?') auch individuell angepasste Detailfragen (bspw. ,Wie kam diese bestimmte Publikation zustande') umfassen.

Durch die Anpassung und Gliederung des Fragenkatalogs auf Basis der forschungsbiografischen Informationen ergibt sich ein chronologischer Aufbau des Leitfadens, der im letzten Abschnitt des Interviews von einigen generalisierenden Fragen abgelöst wird. Zugunsten eines übersichtlichen Leitfadenaufbaus - dessen Gliederungspunkte im Gespräch keine Rolle spielen - werden Aspekte der Themenzyklusmodelle (siehe Kp. 3.2) auf die Akteur*innenebene bezogen. Es ergibt sich der folgende, idealtypische Aufbau eines Leitfadens:

- Latenzphase (das Thema spielt noch keine Rolle für die Person, allerdings existieren bereits inhaltlich verwandte Forschungsprozesse und/oder später relevante Kooperationen)

- Erkennungsphase (die Person betrachtet das Thema erstmals im Kontext der eigenen Forschung als Thema)

- Aneignungsphase (die Person macht das Thema zum Gegenstand der eigenen wissenschaftlichen Arbeit)

- Fokussierungsphase (die einschlägige Forschung trägt Früchte, nimmt aber auch mehr Ressourcen in Anspruch)

- Reorientierungsphase (andere Themenoptionen werden verstärkt geprüft und wahrgenommen, selbst wenn die einschlägige Forschung weiterhin Früchte trägt)

- Gesamtbetrachtung (generelle, retrospektive und zukunftsgerichtete Einschätzungen zur eigenen Themenwahl sowie zur Themenkarriere in der Planungswissenschaft als Ganzes) 
In den jeweiligen Phasen werden sowohl akteur*innenbezogene (bspw. ,Inwiefern mussten Sie sich neues Wissen erarbeiten?') als auch strukturbezogene (bspw. ,Welche Rolle spielte das Thema im Zuge des Berufungsverfahrens?') bzw. dazwischen anzusiedelnde Fragen (, Wie kam Ihre Teilnahme an dem Arbeitskreis zustande?') gestellt.

Die idealtypische Phaseneinteilung der individuellen Beschäftigung mit dem Thema lässt sich mit zwei Ausnahmen auf alle Akteur*innenkategorien anwenden: Nicht bei allen Akteur*innen erlebt das Thema eine Fokussierung, etwa weil strukturell vermittelte Belohnungen ausbleiben. Außerdem tritt nicht bei allen Akteur*innen eine Reorientierungsphase ein, das heißt, das Thema steht zum Ende des Untersuchungszeitraums im Jahr 2014 weiterhin im Mittelpunkt der wissenschaftlichen Tätigkeit. Ebenfalls ist zu berücksichtigen, dass unterschiedliche Interpretationen bezüglich der Frage, wann das Thema angeeignet wurde, möglich sind. Dem methodischen Ausgangspunkt dieser Arbeit, die erstmalige explizite Erwähnung (bspw. „Klimawandel“) und deren Zustandekommen zu betrachten, steht dabei etwa der Verweis auf ein vergleichsweise konstantes Forschungsportfolio, das vor und nach dieser Explizitmachung ähnliche Fragen umfasst (bspw. zur Nachhaltigkeit), gegenüber.

\subsubsection{Auswahl der Interviewpartner*innen und Anonymisierungsstrategie}

Für die Auswahl der Interviewpartner*innen werden aus der Theorie abgeleitete Akteur*innenkategorien definiert, denen mit Hilfe von Datenbankauswertungen passfähige Personen zugeordnet werden. Mit diesem Vorgehen wird die Objektivität der Personenauswahl gewährleistet und es können Erklärungsansätze, die bspw. in den Themenzyklusmodellen der Medien- und Kommunikationswissenschaften aufscheinen (siehe Kp. 3.2), auf den Fall angewendet und geprüft werden.

Um qualitative Informationen aus verschiedenen Blickwinkeln erhalten zu können, müssen die Akteur*innenkategorien unterschiedliche Phasen und Funktionen im Rahmen der Themenkarrieren repräsentieren. Zum einen werden Akteur*innen ausgewählt, die hervorstechende Positionen im Netzwerk einnehmen. Dazu zählen bspw. Akteur*innen, die besonders früh zum Thema veröffentlicht haben, oder solche, die besonders zentral im Ko-Publikationsnetzwerk sind. Zum anderen werden einige Akteur*innen ausgewählt, die eben keine markante Stellung im Netzwerk einnehmen, sondern vielmehr repräsentativ für eine große Gruppe befragt werden können. Auch sind unterschiedliche organisationale 
Zugehörigkeiten - Akteur*innen an Universitäten, Forschungsinstituten sowie im Grenzbereich zwischen Planungswissenschaft und Politik - repräsentiert, um die unterschiedlichen Handlungslogiken erfassen zu können. Insgesamt werden acht Personen aus dem Schrumpfende Städte-Diskurs und neun Personen aus dem Klimawandeldiskurs auf Basis der oben genannten Systematik befragt.

Für die Konzeptualisierung der Akteur*innenkategorien werden Begriffe aus der Theorie der Diffusion von Innovationen nach Rogers (1983 [1962]) aufgegriffen und auf die Entstehung wissenschaftlicher Themen bezogen. Die Übertragung auf die Themenkarrieren Schrumpfende Städte und Klimawandel erfolgt auf Basis des analytischen Rahmens und der Häufigkeiten einschlägiger Artikel, aus denen sich ein Aufmerksamkeitsverlauf ermitteln lässt. Dieser besteht aus Latenz-, Durchbruch-, Fokus- und Normalisierungsphase. Die ursprünglichen Kategorien nach Rogers - innovators, early adopters, early majority, late majority und laggards (Rogers 1983 [1962]: 247) - werden dabei modifiziert: ,Innovators ' werden zu ,Pionier*innen', weil nicht Innovationen, sondern Themen untersucht werden und um zu betonen, dass die vereinzelten einschlägigen Vorarbeiten nicht zwangsläufig in einem Durchbruch münden. Da anstatt der Glockenkurve nach Rogers (siehe Abb. 6.5) ein abrupter Durchbruch der Aufmerksamkeit gefolgt von einer Fokusphase mit zwei Höhepunkten (siehe Abb. 6.6) identifiziert wurden, werden early majority und late majority zu Follower*innen zusammengefasst und die Abgrenzungen angepasst (für Erläuterungen zu den einzelnen Phasen und Akteur*innenkategorien siehe Kp. 7, 8 und 9.1).

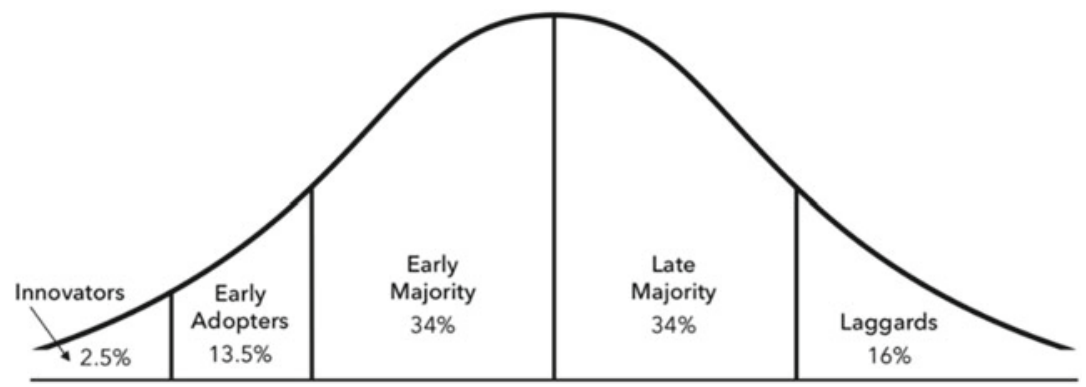

Abb.6.5 „Adopter categorization on the basis of innovativeness“ (Quelle: Rogers (1983 [1962]: 247)) 


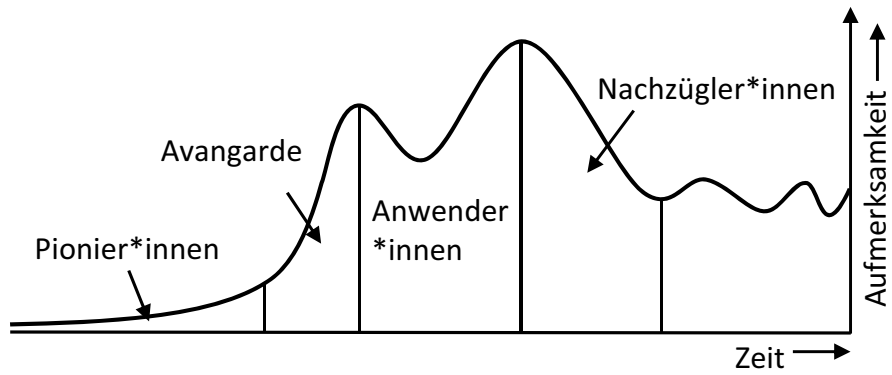

Abb.6.6 Adopter-Kategorien auf Basis der wissenschaftlichen Themenkarriere (Eigene Darstellung)

Für die Auswahl der Interview-Partner*innen, werden die folgenden Akteur*innenkategorien festgelegt:

- Pionier*in (Person, die in der Latenzphase einschlägig veröffentlicht)

- Avantgardist*in (Person, die in der Durchbruchsphase erstmals einschlägig veröffentlicht)

- Anwender*in (Person, die in der Fokusphase erstmals einschlägig veröffentlicht)

- Nachzügler*in (Person, die nach der Fokusphase erstmals einschlägig veröffentlicht)

- Autorität (Person, die besonders häufig einschlägig zitiert wird)

- Fördermittelgeber*in (Person, die an Entscheidungen der Fördermittelvergabe beteiligt ist, die im Grenzbereich zwischen Planungswissenschaft und Politik anzusiedeln und für die Entwicklung des Themas relevant sind)

Indem Autoritäten und teilweise auch Fördermittelgeber*innen $\mathrm{zu}$ einem bestimmten Zeitpunkt erstmals einschlägig publizieren, können einige Personen mehreren Akteur*innenkategorien zugeordnet werden (bspw. Pionier*in und Autorität). Auch ist möglich, dass eine Person in beiden Themenkarrieren eine Rolle einnimmt.

Auf Basis der Kategorien werden Vorauswahlen von Personen durch Datenbankauswertungen getroffen, aus denen Personen nach dem Zufallsprinzip ausgewählt werden. Kommt ein Interview nicht zu Stande, wird eine andere Person nach dem genannten Verfahren ausgewählt. 
Im Zuge der Beschreibung der beiden Themenkarrieren in Kapitel 7 und 8 sollen möglichst tiefe und unverfälschte Einblicke in das empirische Material gegeben werden. Hierbei nimmt die Wiedergabe von Interviewaussagen eine wichtige Funktion ein, sodass sie nicht nur in paraphrasierter Form, sondern auch als direktes Zitat in den Text einfließen. Diese Zitate sind nicht nur aussagekräftig im Hinblick auf die Beantwortung der Fragestellung, sondern sie vermitteln einige Mechanismen der Themenwahl auch besonders anschaulich. Indem die Leser*innen einen Bezug zu den in den Zitaten geschilderten Situationen entwickeln können, lassen sich einige Aspekte prägnanter darstellen, als etwa mit Hilfe abstrakter Theorierückgriffe oder quantitativer Auswertungen.

Allerdings ist dem Schutz der Quellen oberste Priorität einzuräumen, insbesondere angesichts der vergleichsweise kleinen Fachgemeinschaften, die sich um die beiden Themen gruppieren. Es sind also einige Maßnahmen erforderlich, um die Anonymität der Interviewten zu gewährleisten. Dabei sollen einerseits so wenig Informationen wie möglich Rückschlüsse auf den*die Interviewte*n zulassen, sodass der Kreis möglicher Urheber*innen maximiert wird. Gleichzeitig sollen aber so viele Informationen wie nötig angegeben werden, um die Nachvollziehbarkeit und Aussagekraft des Zitats durch ihre Kontextualisierung sicherzustellen. Die jeweils sprechende Person wird daher nicht in Form eines Schlüssels angegeben, da die Aneinanderreihung der Zitate auf der Grundlage dieses Schlüssels den Kreis der möglichen Urheber*innen weiter verkleinern könnte. Um die Interviews dennoch als Quelle zu markieren, werden stattdessen die einzelnen Aussagen von $i 1$ bis $i 68$ durchnummeriert, wobei direkt aneinanderanschließende Aussagen desselben Interviews die gleiche Nummer erhalten. Namen, Geschlechter, Orte, Organisationen und ähnliche Hinweise auf die Quelle werden aus der Aussage entfernt und wo nötig durch Allgemeinplätze ersetzt. Die exemplarische Darstellung der research trails (siehe Abb. 7.2 und Abb. 8.4) erfolgt außerdem nicht auf der Grundlage der Forschungsbiografien tatsächlich interviewter Personen. In den Fällen, in denen trotz dieser Maßnahmen ein zu geringer Spielraum für die Anonymisierung besteht, muss auf die Aussagen verzichtet werden oder die betreffende Person um die Freigabe der Passagen gebeten werden.

\subsubsection{Durchführung und Auswertung der Interviews}

Insgesamt wurden acht Personen aus dem Schrumpfende Städte-Diskurs und neun Personen aus dem Klimawandeldiskurs auf Basis der oben erläuterten Systematik befragt. Die Dauer der Interviews lag, abhängig von der Akteur*innenkategorie und Verfügbarkeit der Person, zwischen 40 und 120 Minuten. Die Interviews 
wurden nach Möglichkeit face-to-face, in sieben Fällen aber auch telefonisch durchgeführt. Während die Erhebung und Auswertung forschungsbiografischer Daten im Vorfeld aller Interviews angewendet wurde, wurde der Einsatz einer großformatig ausgedruckten, visualisierten Forschungsbiografie während des Gesprächs nur in neun Interviews für sinnvoll erachtet und umgesetzt. Für die Wahl dieser Methode war ausschlaggebend, ob durch sie ein Mehrwert bezüglich der im Gespräch zu erwartenden Erkenntnisse entsteht. Spielte die Erforschung des Themas bspw. nur eine geringe und zeitlich stark begrenzte Rolle in der nicht immer nur wissenschaftlichen - Karriere der Akteur*innen, so wurde von dem Einsatz der Abbildung im Gespräch abgesehen.

Durch die Vorab-Auswertung der forschungsbiografischen Daten konnte auch unabhängig von ihrer Visualisierung und Vorlegung im Interview - die Erinnerung der befragten Person stimuliert werden oder Fehlerinnerungen im Dialog aufgeklärt werden, insbesondere wenn es um die zeitliche Einordnung bestimmter Handlungen ging. Indem die befragten Personen die intensive Vorarbeit des Interviewers sowie die in der Struktur der Wissenschaft angelegte Überprüfbarkeit vieler Aussagen registrierten, wurden bewusste Verfälschungen, Übertreibungen oder Auslassungen seitens der interviewten Personen von vornherein auf ein Minimum reduziert.

Kam die forschungsbiografische Abbildung zum Einsatz, wurde sie einleitend zu Beginn des Interviews vorgelegt und diente der beiderseitigen zeitlichen und sachlichen Orientierung während des Gesprächs. Sie fungierte als Stimulus und Leitfadenergänzung und ermöglichte einen unmittelbaren Einstieg in weit zurückliegende Abschnitte der jeweiligen Forschungsbiografie. Die kompakte Darstellung komplexer Daten machte Veränderungen, Brüche und Kontinuitäten einfach nachvollziehbar, sodass sie voraussetzungsfrei als Angelpunkt der Gesprächsführung dienen konnte. Hierbei konnten sich beide Gesprächspartner*innen immer wieder auf Details der Biografie, Zusammenhänge zwischen einzelnen Elementen sowie auch auf das Fehlen wichtiger Informationen beziehen (Aljets 2015: 116). Ein letztes, wichtiges Argument für den Einsatz der research trails ist, dass die intensive Vorarbeit des Interviewers bei Gesprächsbeginn offenkundig war, sodass die Interviewten motiviert wurden, das Ihrige zu einem fokussierten, offenen und fruchtbaren Gespräch beizutragen.

Einzelfallabhängig wurde für den Einstieg entweder eine biografisch informierte Frage gewählt - bspw. , Sie haben sich in ihrer ersten Publikation mit der Bewältigung von Umweltkatastrophen auseinandergesetzt, wie kam es dazu? - oder es wurde mit der weniger vorstrukturierten, fallunabhängigen Frage ,Können Sie sich an den Moment erinnern, als Sie das Thema ,Schrumpfung ‘ zum ersten 
Mal wahrgenommen haben? ' begonnen. Von hier ausgehend entfaltete jedes Interview eine eigene Dynamik, die sich unterschiedlich stark an dem Leitfaden und dem research trail orientierte. Unabhängig von deren wechselnden Bedeutung hat sich in jedem Interview die intensive Vorarbeit bezahlt gemacht, etwa wenn der interviewten Person mit Jahreszahlen oder Publikationstiteln assistiert werden musste oder wenn Unstimmigkeiten in den Erzählungen direkt angesprochen und aufgeklärt werden konnten.

Die primäre Aufgabe des Interviewers im Gespräch war es, zu gewährleisten, dass die Fokussierung auf die im Sinne der Fragestellung relevanten Informationen beibehalten wurde und dass die zentralen Fragen des Leitfadens besprochen wurden. Zudem musste der Interviewer nicht nur inhaltlich (s.o.), sondern auch emotional aufmerksam sein, um etwa intervenieren zu können, wenn die befragte Person mit einer ehrlichen Antwort haderte. Ein weiterer wichtiger Punkt war es, zu vermitteln, dass die genannten Aussagen anonymisiert werden, sodass ein sicherer Raum geschaffen wurde, in dem offen gesprochen werden konnte.

Der entscheidende Faktor dafür, dass die Informationsgewinnung im Rahmen der Interviews ausgesprochen erfolgreich verlaufen ist, war die hohe Kooperations- und Auskunftsbereitschaft der Interviewten. Hierfür war zum einen die intensive Vorbereitung durch den Interviewer ein wichtiger Faktor, der die Motivation und Fokussierung insbesondere zu Beginn des Gesprächs erkennbar erhöhte. Vor allem aber ist an dieser Stelle auf die hohe Bereitschaft der Interviewten hinzuweisen, Zeit und Anstrengungen zu investieren, um in teilweise weit zurückliegende Situationen einzutauchen und tatsächliche Faktoren der Themenwahlen zu identifizieren. Es entstand der Eindruck, die Akteur*innen würden die erstmalige oder zumindest seltene Gelegenheit, auf informierter Basis über ihr alltägliches Handeln zu reflektieren, dankbar annehmen. Die durchgeführten Interviews lassen sich daher beschreiben als ein jeweils ein- bis zweistündiges intensives und gemeinsames Suchen nach den tatsächlichen Faktoren und Zusammenhängen der jeweiligen Themenwahlen unter Zuhilfenahme der vorab erhobenen und aufbereiteten forschungsbiografischen und bibliografischen Informationen und mit der größtmöglichen Offenheit und Ehrlichkeit.

Zur Vorbereitung der computergestützten Interviewauswertung wurden die Interviews aufgezeichnet, vollständig transkribiert und codiert. In zwei Interviews war keine durchgängige Audio-Aufnahme möglich, sodass die Transkription hier auf der Basis händischer Mitschriften erfolgte. Die Erhebung und Auswertung der Daten erfolgte iterativ, das heißt, jeder Erhebungs-, Auswertungs- und Analyseprozess war durch die vorhergehenden Forschungsprozesse informiert und bildete die Grundlage für weitere Forschungsprozesse. Beispielsweise wurden in wenigen Fällen Detailfragen zur Validierung anderer Interviewaussagen ergänzt. 
Bei der Interpretation der Interviewdaten waren die in Abschnitt 6.4.1 genannten methodischen Herausforderungen zu berücksichtigen. Insoweit sich die für die Beschreibung der beiden Themenkarrieren verwendeten Aussagen auf manifeste Handlungen und Objekte beziehen - bspw. Veröffentlichungen oder Vorträge und ihre zeitliche Einordnung -, wurden diese überprüft. Zudem wurden einige weitere Personen zur Überprüfung und Klärung von Interview-Aussagen mit jeweils spezifischen Detailfragen konfrontiert. War eine Validierung nicht möglich, wurde auf die Verwendung der Aussage verzichtet oder auf ihre Unüberprüfbarkeit hingewiesen.

Nicht nur Aussagen aus den Interviews flossen in die Analyse der Themenkarrieren ein. Zum einen wurden Detailaussagen weiterer Akteur*innen verwendet, die zur Interpretation der Netzwerkanalyse und der Interviews sowie zum Verständnis spezifischer Prozesse der Themenkarrieren gezielt erhoben wurden. Zum anderen wurde auf Ausführungen zurückgegriffen, die in Workshops und Konferenzen im Kontext des TIPKUS-Projektes getätigt wurden. Insbesondere die forschungsbiografischen Reflexionen im Rahmen des TIPKUS Projekt-Symposiums „Themenkarrieren in der Planungswissenschaft“ am 07.02.2018 an der TU Dortmund sind hier zu nennen. Dabei reflektierten Hans Heinrich Blotevogel, Christina von Haaren, Johann Jessen, Heiderose Kilper und Walter Siebel ${ }^{5}$ im Rahmen von Vorträgen über ihre eigene Forschungsbiografie, jeweils verbunden mit der Frage, wie und warum sich bestimmte Themen zu eigenen Forschungsschwerpunkten entwickelt haben.

Open Access Dieses Kapitel wird unter der Creative Commons Namensnennung 4.0 International Lizenz (http://creativecommons.org/licenses/by/4.0/deed.de) veröffentlicht, welche die Nutzung, Vervielfältigung, Bearbeitung, Verbreitung und Wiedergabe in jeglichem Medium und Format erlaubt, sofern Sie den/die ursprünglichen Autor(en) und die Quelle ordnungsgemäß nennen, einen Link zur Creative Commons Lizenz beifügen und angeben, ob Änderungen vorgenommen wurden.

Die in diesem Kapitel enthaltenen Bilder und sonstiges Drittmaterial unterliegen ebenfalls der genannten Creative Commons Lizenz, sofern sich aus der Abbildungslegende nichts anderes ergibt. Sofern das betreffende Material nicht unter der genannten Creative Commons Lizenz steht und die betreffende Handlung nicht nach gesetzlichen Vorschriften erlaubt ist, ist für die oben aufgeführten Weiterverwendungen des Materials die Einwilligung des jeweiligen Rechteinhabers einzuholen.

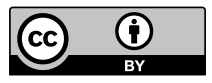

\footnotetext{
${ }^{5}$ Walter Siebel legte seine Reflexion schriftlich vor, nachdem er den mündlichen Vortrag kurzfristig absagen musste.
} 